\title{
Critical tests of stellar evolution in open clusters
}

\section{Stellar population and dynamical evolution of IC $4651^{\star, \star \star}$}

\author{
S. Meibom ${ }^{1,2}$, J. Andersen ${ }^{1}$, and B. Nordström ${ }^{1,3}$ \\ 1 Astronomical Observatory, Niels Bohr Institute for Astronomy, Physics, and Geophysics, \\ Juliane Maries Vej 30, 2100 Copenhagen, Denmark \\ e-mail: ja@astro.ku.dk \\ 2 Astronomy Department, University of Wisconsin, Madison, WI 53706, USA \\ 3 Lund Observatory, PO Box 43, 22100 Lund, Sweden \\ e-mail: birgitta@astro.lu.se
}

Received 28 November 2001 / Accepted 30 January 2002

\begin{abstract}
We present multiple-epoch radial-velocity observations for 104 stars in a $10^{\prime} \times 10^{\prime}$ field of the intermediate-age open cluster IC 4651 to $V \sim 14.5$. Only 13 stars (13\%) of the full sample are field stars. From the 44 single member stars we find a mean radial velocity of $-30.76 \pm 0.20 \mathrm{~km} \mathrm{~s}^{-1}$, and the 12 single red-giant members yield a true radial-velocity dispersion of $0.74 \mathrm{~km} \mathrm{~s}^{-1}$. Of the 19 giant members, $7(37 \%)$ are spectroscopic binaries with periods up to 5000 days, while $35(52 \%)$ of the 67 main-sequence and turnoff members are binaries with periods less than $\sim 1000$ days. Combined with our deep, accurate CCD Strömgren photometry in a $\sim 21^{\prime} \times 21^{\prime}$ field of IC 4651 (Meibom 2000), these data substantially improve the definition of the cluster locus in the colour-magnitude diagram and the spatial structure of the cluster, although the photometry shows that IC 4651 contains at least twice as many stars on the upper main sequence as was believed when the radialvelocity survey was initiated. The single cluster members define a very tight sequence in the CMD, and two sets of isochrones from stellar models with convective overshooting $\left(d / H_{\mathrm{p}}=0.2\right)$ have been fit to it. Our best estimate for the age of IC 4651 is $1.7 \pm 0.15 \mathrm{Gyr}$, assuming $[\mathrm{Fe} / \mathrm{H}]=0.12$ (Hyades) and $E_{(b-y)}=0.071$. Including the $\sim 650$ stars newly discovered from the photometry, we estimate the present total mass of IC 4651 to be $\sim 630 M_{\odot}$, excluding any undetected stellar remnants. The corresponding tidal cutoff radius is $\sim 22^{\prime}$. IC 4651 shows evidence of moderate mass segregation: Most of the turn-off stars and nearly all the red giants are located at radii smaller than $\sim 7^{\prime}$, while the lower main-sequence stars are less centrally concentrated. The spatial distributions of cluster and field stars indicate that additional cluster stars are probably still to be found outside the fields studied so far. Comparison of the present mass function of IC 4651 with plausible initial mass functions indicates that the cluster initially contained at least $\sim 8300$ stars with a total mass of $\sim 5300 M_{\odot}$. Thus, of the original cluster stars only $\sim 7 \%$, containing $\sim 12 \%$ of the initial mass, remain today. Of the initial cluster mass, $\sim 35 \%$ has been lost due to evolution of the most massive stars into white dwarfs or other remnants while the remaining $\sim 53 \%$, comprising $\sim 93 \%$ of the original low-mass stars, appear to have migrated out of the observed field or been lost from the cluster altogether. IC 4651 is currently $1 \mathrm{kpc}$ closer to the Galactic center than its "sister" cluster NGC 3680 (Nordström et al. 1997), but their Galactic orbital parameters indicate that the mean orbital radius of IC 4651 is in fact larger by $0.7 \mathrm{kpc}$, providing a plausible reason why it is much less advanced in its dynamical evolution than the coeval cluster NGC 3680.
\end{abstract}

Key words. open clusters and associations: IC 4651 - stars: HR diagram - stars: evolution - stars: kinematics stars: fundamental parameters

Send offprint requests to: S. Meibom, e-mail: meibom@astro.wisc.edu

* Based on observations obtained with the Danish 1.5-m telescope at the European Southern Observatory, La Silla, Chile.

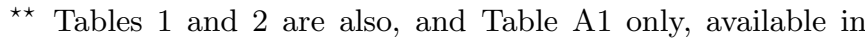
electronic form at the CDS via anonymous ftp to cdsarc.u-strasbg.fr (130.79.128.5) or via http://cdsweb.u-strasbg.fr/cgi-bin/qcat?J/A+A/386/187

\section{Introduction}

Open star clusters are a rich source of information on several key topics in stellar and galactic astrophysics. First, stars seem to form primarily in clusters. Second, as the stars evolve, the cluster sequence in the colourmagnitude diagram (CMD) traces the form of progressively older isochrones in considerable detail, and thus 
allows to check the predictions of stellar evolution models. Third, rich clusters can be observed to large distances from the Sun and, therefore, serve as probes of the age and abundance structure of the galactic disk. And fourth, as clusters evolve through dynamical interactions, internally and with the Galactic tidal field, their structure also changes and the cluster stars are gradually lost to the field. The many uses of (especially older) open clusters in stellar and Galactic astrophysics were comprehensively reviewed by Friel (1995) and are illustrated, e.g., by Friel \& Janes (1993) and Janes \& Phelps (1994).

The key observational tool in the study of a star cluster is an accurate CMD in a well-calibrated colour system. However, any determination of distance, reddening, chemical composition, or age, and any detailed isochrone fit, assumes that one can distinguish cluster members from field stars and identify binary and multiple stars among the cluster members. Further, in studies of the dynamical evolution of clusters one also needs information on the velocities and masses of the individual (single or binary) cluster members. Detailed membership information can be provided by proper motion and radial velocity data, the latter also allowing to detect spectroscopic binaries if multiple observations are obtained over long intervals.

The depth of analysis that can be achieved with complete, high-quality photometric and kinematic data is perhaps best illustrated in the classic study of the Hyades by Perryman et al. (1998), while the observational history of NGC 3680 demonstrates the difficulty in reaching firm conclusions without such information (Nordström et al. 1997). Yet, besides these two clusters, the Pleiades (Raboud \& Mermilliod 1998a), Praesepe (Raboud \& Mermilliod 1998b), NGC 752 (Daniel et al. 1994), and M67 (Nissen et al. 1987; Mathieu et al. 1986) are still the only clusters to have reasonably complete kinematic data. The present paper adds the intermediate-age cluster IC 4651 (age 1.7 Gyr) to that select company.

Our study of IC 4651 and its "sister" cluster NGC 3680 (Nordström et al. 1997), of nearly identical chemical composition and age, was initiated in 1988 to provide tighter observational constraints on stellar models and on ages derived from them. Indeed, previously published ages for the relatively nearby IC 4651 ranged from 1.3 Gyr (Mazzei \& Pigatto 1988) to 4.0 Gyr (Maeder 1990), even when based on the same photometry. Moreover, both clusters were used as evidence both for and against models with extended convective cores ("overshooting" models). Since then, evidence from NGC 3680 (see Nordström et al. 1997) and many other sources has proved the reality of "overshooting" beyond doubt; the debate now concerns its physical nature and quantitative description.

However, our study of NGC 3680 not only ruled out the use of standard models for stars above $\sim 1.2 M_{\odot}$, it also showed NGC 3680 to be an extreme case of dynamical evolution: Virtually all cluster stars more than two magnitudes below the turnoff have been lost from the area studied so far, and of the remainder some $60 \%$ are spectroscopic binaries. Clearly, neither stellar evolution nor, especially, Initial Mass Functions (IMF's) can be studied in open clusters without explicit consideration of their dynamical evolution, as emphasized also in recent theoretical studies (de la Fuente Marcos 1997; Portegies Zwart et al. 2001, and references therein).

The present study of IC 4651 underscores this point again, based on the recent, accurate Strömgren uvby photometry for over 17000 stars in a $\sim 21^{\prime} \times 21^{\prime}$ field around IC 4651 by Meibom (2000); references to previous work on the cluster can be found in that paper. Meibom (2000) succeeded in delineating the main sequence down to six magnitudes below the turnoff; even more importantly, it was shown that the cluster covers at least twice the area and contains twice as many stars as previously believed. Thus, the radial-velocity survey presented in the present paper turns out in fact to only cover the central, classical cluster area, but it remains adequate to address the fundamental dynamical properties of IC 4651. We note that a radial-velocity study of the red giant stars in IC 4651 (and NGC 3680) was published already by Mermilliod et al. (1995), but these stars are included again here, because additional observations and an improved zero-point calibration have become available after that time.

In the following, we describe the new observational data for IC 4651 in Sect. 2 and analyze them in Sect. 3 in terms of cluster membership, binary star detection, and field star contamination. Section 4 is devoted to a comparison of theoretical isochrones with the cluster sequence, while Sect. 5 discusses the current structure and dynamical state of the cluster and compares with theoretical computations. Finally, Sect. 6 summarizes our findings and discusses the outlook for further studies.

\section{Observational data for IC 4651}

\subsection{Photometry}

The new CMD for IC 4651 by Meibom (2000), based on CCD uvby photometry for 17640 stars to $V \sim 21^{\mathrm{m}}$ in a $\sim 21^{\prime} \times 21^{\prime}$ field supersedes all previous photometry for the main-sequence region of the cluster. All details of the observations and reduction procedures are given in the paper, which also summarizes the observational history of IC 4651. Typical standard errors of the mean magnitudes are $0{ }^{\mathrm{m}} 007$ in $V, 0{ }^{\mathrm{m}} 007$ in $b, 0{ }^{\mathrm{m}} 009$ in $v$, and $0{ }^{\mathrm{m}} 010$ in $u$.

The salient findings from the photometry were: $(i)$ : a greatly superior definition of the main sequence in the $(v-y)-V \mathrm{CMD}$, as compared to the traditional combination $(b-y)-V ;(i i)$ : an effective doubling of the known size of the cluster in terms of both area and number of stars; and (iii): a clear tendency for the faint main-sequence stars to prefer the outskirts of the cluster. All three topics are further discussed below.

While the Meibom (2000) paper was in press, a new CMD comprising uvby- $\mathrm{H}_{\beta}$ photometry of 387 stars in a $13.5^{\prime} \times 13.5^{\prime}$ field to $V \sim 16^{\mathrm{m}}$ was published by AnthonyTwarog \& Twarog (2000). Comparison with our data shows a similar colour discrepancy for the red giants 
$\left(V<11^{\mathrm{m}} 5,(b-y)>0.6\right)$ as found by Meibom (2000, Fig. 2) relative to the earlier data by Anthony-Twarog \& Twarog (1987), our photometry being about $0.03 \mathrm{mag}$ brighter in $V$ and bluer in $b-y$ than theirs. This is readily understandable because our observations were calibrated primarily for the main-sequence region; also, the uvby colour calibrations are less reliable for red giants, both theoretically and observationally. For the 252 stars in the colour range covered by our standard stars, i.e., $(b-y)<$ 0.6 , the average differences are $0.000 \pm 0.026$ (s.d.) in $V$ and $0.001 \pm 0.026$ (s.d.) in $(b-y)$, respectively, a quite satisfactory result.

Given the close agreement between the photometry by Anthony-Twarog \& Twarog (2000) and Meibom (2000), but also the larger area and greater depth of the latter, we will use the Meibom photometry in the remainder of this paper.

\subsection{Radial velocities}

In order to distinguish between field and cluster stars and detect the spectroscopic binaries in the cluster, radialvelocity observations were made during the years 19891997 with the photoelectric scanner CORAVEL (Mayor 1985) on the Danish 1.54-m telescope at ESO, La Silla. The observations are referred to the accurate velocity zero-point determined from a large number of observations of minor planets and standard stars by Udry et al. (1999). The observing list comprised all known red giants in and near the cluster, plus all candidate main-sequence and turnoff stars brighter than the limiting magnitude of CORAVEL $(B \sim 15)$ from the then largest known photometric surveys of the cluster, by Eggen (1971) and Anthony-Twarog et al. (1988, AMCT).

With the aim to obtain three or more observations per star in IC 4651, a total of 520 observations were made of the 117 programme stars, including about 220 measurements of the 20 red giants and 300 measurements of 97 turnoff and main-sequence stars. Many of the stars in the latter group rotate rapidly, so high signal levels were required and typical integration times were of the order of an hour; for the red giants integration times were 5-10 min. Only 13 stars, mainly in the turn-off region, rotated too fast for a proper determination of their radial velocity; in the system of Meibom (2000), these stars are MEI 5489, 7386, 7628, 10059, 10232, 11035, 11305, 11852, 11888, 12035, 12089, 13015, and 14069 .

If a first observation of a photometric non-member gave a velocity far from the cluster mean, or a second observation immediately established a star as an obvious spectroscopic binary, observations were normally stopped. Otherwise, three or more observations were obtained over a time interval of typically 1000-2000 days for the mainsequence stars, and 5000 days for the red giants (observed since 1983). The red giant data were analyzed by
Mermilliod et al. (1995), but are rediscussed here because additional observations have been obtained.

The results of the radial-velocity observations are summarized in Table 1. Star numbers are given in the systems of Meibom (2000, MEI), Eggen (1971, E, numbers less than 200) and Anthony-Twarog et al. (1988, AT, numbers larger than 1000). A few outlying red cluster giants have numbers from either Lindoff (1972, L, Nos. 241 and 244) or Mermilliod et al. (1995) (M, Nos. 811, 812, and 817). Following the mean velocity $\langle R V\rangle$ and its error, $\sigma_{<V\rangle}$, $n$ is the number of observations and $\sigma_{1}$ the standard deviation of a single observation; for double- or triple-lined binaries, we give the systemic velocity and $\sigma_{<V>}$ as computed with the method of Wilson (1941). O/C is the ratio $\sigma_{1} / \sigma_{\mathrm{C}}$, where $\sigma_{\mathrm{C}}$ is the average value of the error estimated for each observation of the star from the number of counts and the shape of the profile. The algorithm for computing $\sigma_{\mathrm{C}}$ has been improved since the study of NGC 3680 by Nordström et al. (1996), and no dependence of O/C on stellar rotation is seen in IC 4651. Consequently, no corrections have been applied to the present data. If $\mathrm{O} / \mathrm{C}<1$, $\sigma_{<V>}=\sigma_{\mathrm{C}} / n^{1 / 2}$ is given as a more realistic error estimate than $\sigma_{1} / n^{1 / 2}$. Finally, $\Delta T$ is the time span (days) covered by the observations; the median value of $\Delta T$ is 1845 days.

In order to facilitate future studies of the velocity variables, the individual radial velocity observations of all stars are provided in Table A1 (in electronic form only). Figure 1 shows the CMD of IC 4651, with the stars with radial-velocity data identified by squares. The small points indicate stars not included in the radial-velocity survey, either because they were too faint or because they lie outside the inner field believed to contain the cluster when the radial-velocity survey was conducted. The many new outlying and/or faint cluster members would form ideal targets for a new survey with a multi-fiber spectrometer on a large telescope. The distribution of the observed mean radial velocities is shown in Fig. 2a; as seen, the velocities of the (relatively few) field stars are distributed in the range -80 to $+40 \mathrm{~km} \mathrm{~s}^{-1}$.

\subsection{Notes on individual stars}

A few stars require separate comment. First, by mistake, Table 2 of Meibom (2000,MEI) identified MEI 6726 with both E73 and AT 1230; as indicated in our Table 1, E73 is in fact the star MEI 7036. Moreover, correction of a previously misidentified observation shows that MEI 6333 is in fact a single star, contrary to the assignment of variability by Mermilliod et al. (1995).

More substantially, additional radial velocities have been obtained for most of the red giant stars since Mermilliod et al. (1995), extending the time basis of the data. These new data led to the detection of two new spectroscopic binaries amongst the red giants (MEI 11218 and 14527). We have also recomputed the spectroscopic binary orbits given previously by Mermilliod et al. (1995). The resulting orbital elements are given in Table 2 and 


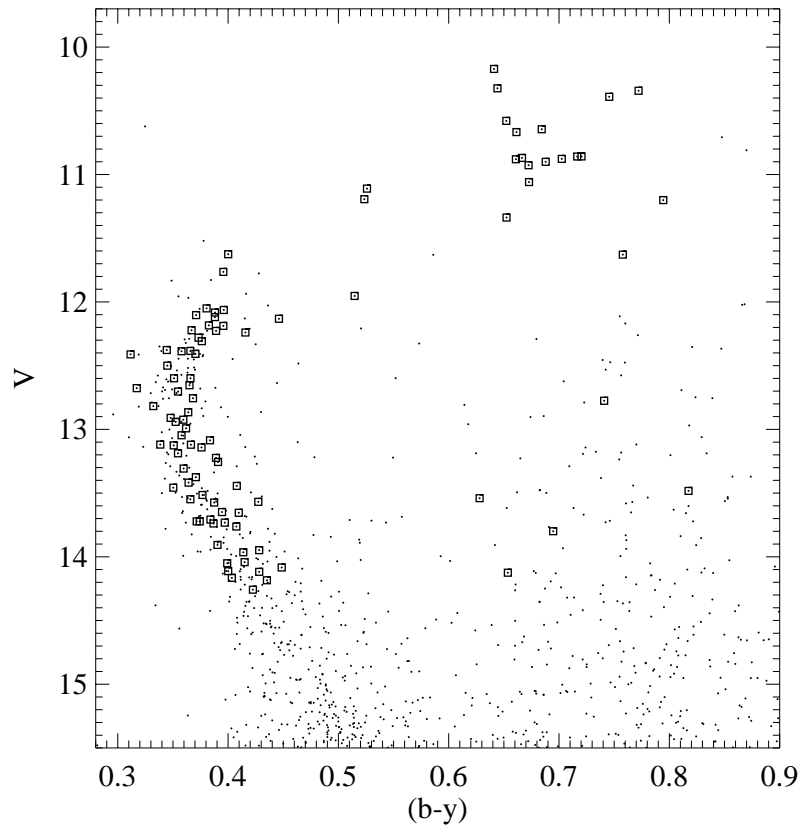

Fig. 1. The $(b-y, V)$ CMD of IC 4651 (Meibom 2000). Stars with radial-velocity data are framed with squares.

are significantly improved in accuracy over those by Mermilliod et al. (1995).

For double-lined binaries with insufficient data for an actual orbit determination, the Wilson (1941) method also provides an estimate of the mass ratio $q=M_{\mathrm{B}} / M_{\mathrm{A}}$. For the stars labeled "sb2" in Table 1, we find the following results: MEI 6726: $q=0.87 \pm 0.03$; MEI 6733: $q=0.75 \pm 0.25$; MEI 8302: $q=0.49 \pm 0.08$; MEI 9278: $q=0.85 \pm 0.09$; and MEI 10029: $q=0.90 \pm 0.20$. MEI 10019 and MEI 11504 show double lines, but only one component varies in velocity; thus, these objects are likely to be triple systems and no mass ratio can be determined.

\subsection{Rotational velocities}

Rotational velocities were determined for the stars in IC 4651 as described by Benz \& Mayor $(1981,1984)$ and are included, with their errors, in Table 1 . The mean rotational velocity of the 31 measurable single main-sequence stars is $18.8 \pm 2.4 \mathrm{~km} \mathrm{~s}^{-1}$ (s.e.m.), with a standard deviation of $13.1 \mathrm{~km} \mathrm{~s}^{-1}$. For comparison, the single mainsequence stars in NGC 3680 have a mean rotational velocity of $15.3 \pm 3.1 \mathrm{~km} \mathrm{~s}^{-1}$ (s.e.m.) with a standard deviation of $13.3 \mathrm{~km} \mathrm{~s}^{-1}$, essentially the same as in IC 4651 . The modest sample sizes do not allow further detailed study of, e.g., the orientation of rotational axes, etc.

\section{The stellar population of IC 4651}

\subsection{Spectroscopic binary detection}

As discussed in detail in Nordström et al. (1997), comparison of the observed and expected standard deviations of multiple radial-velocity observations of a star
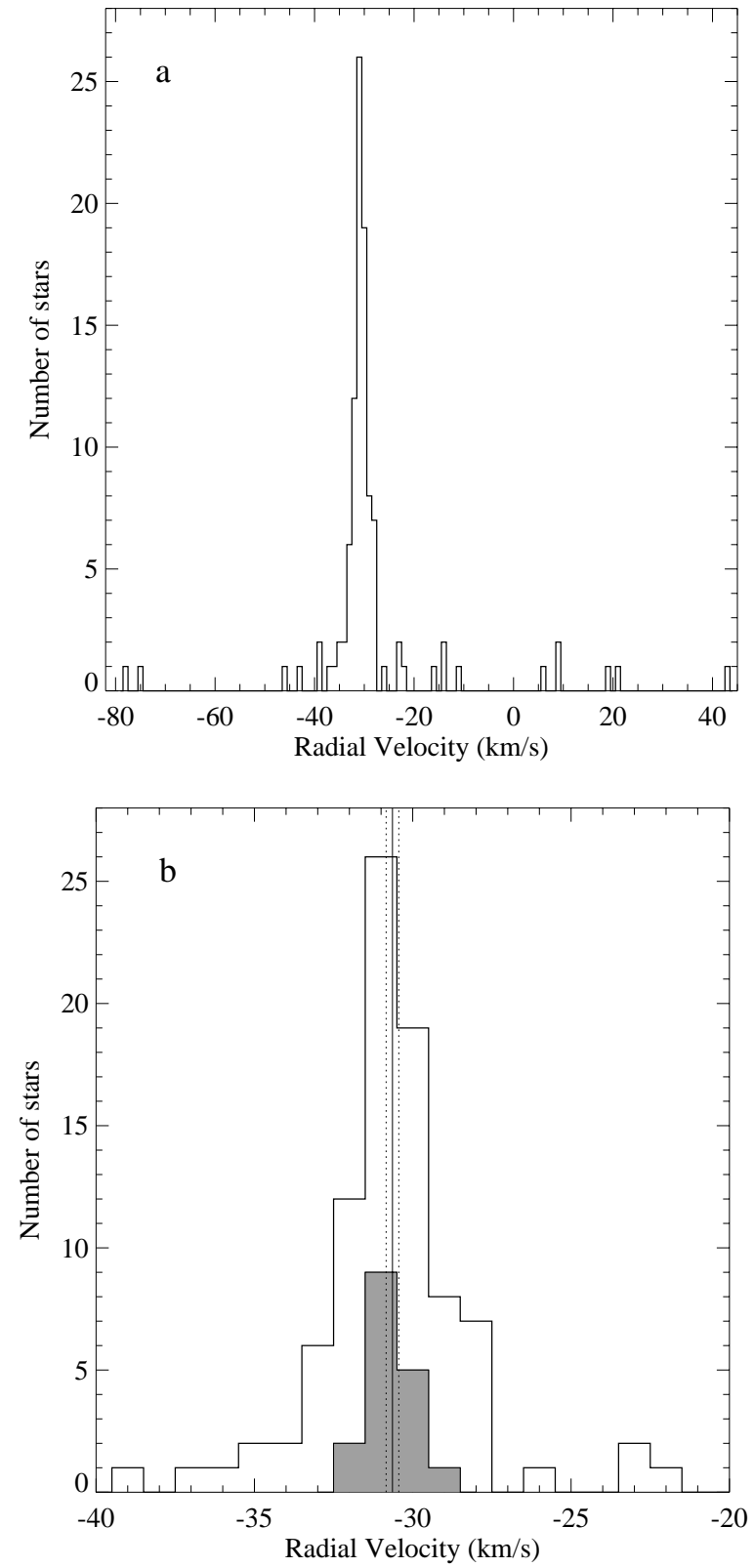

Fig. 2. Radial-velocity histograms for a) all observed stars; b) radial velocity members (line) and red giant members only (filled curve). The vertical lines show the mean cluster velocity and its mean error $\left( \pm 1 \sigma_{<V>}\right)$.

allows to compute the probability $P\left(\chi^{2}\right)$ that the velocity is constant, i.e. that the star is single. $P\left(\chi^{2}\right)$ is given in Table 1 for all programme stars with at least 2 observations. Consistent with other studies using CORAVEL data, we adopt the conservative variability/duplicity criterion $P\left(\chi^{2}\right)<0.01$ to distinguish the certain binaries from the probable single stars in Table 1. A few binaries were identified by a double correlation peak in just a single observation. Our detection efficiency decreases rapidly for binaries with periods longer than our typical $\Delta T(\sim 2000$ days $)$ and for small velocity amplitudes, so undetected binaries certainly remain in IC 4651. 
Table 1. Mean radial and rotational velocities, with errors (all $\mathrm{km} \mathrm{s}^{-1}$ ), and duplicity and membership diagnostics $P\left(\chi^{2}\right)$ and $P(R V)$ for 104 stars in IC 4651. "Other" numbers are: E: Eggen (1971), A: Anthony-Twarog et al. (1988), L: Lindoff (1972), and M: Mermilliod et al. (1995). Remark codes are: "M": star discussed by Mermilliod et al. (1995); O: new orbit in Table 2; sb2/3: double/triple-lined spectroscopic binary; pm: probable member; *: remark in Sect. 2.3.

\begin{tabular}{|c|c|c|c|c|c|c|c|c|c|c|c|c|c|c|}
\hline MEI & Other & $V$ & $b-y$ & $<R V>$ & $\sigma_{<V>}$ & $n$ & $\sigma_{1}$ & $\mathrm{O} / \mathrm{C}$ & $\Delta T$ & $P\left(\chi^{2}\right)$ & $P(R V)$ & $v \sin i$ & $\sigma_{v}$ & Rem. \\
\hline 5289 & E1 & 12.91 & 0.342 & -29.84 & 1.49 & 3 & 2.26 & 0.88 & 1875 & 0.471 & 0.657 & 36.3 & 5.7 & \\
\hline 5456 & $\mathrm{E} 2$ & 13.46 & 0.344 & -38.55 & 2.01 & 3 & 3.49 & 2.21 & 1107 & 0.008 & 0.002 & 26.9 & 2.7 & \\
\hline 5658 & E102 & 12.10 & 0.370 & -36.77 & 10.70 & 4 & 21.41 & 9.78 & 2887 & 0.000 & 0.578 & 34.0 & 3.4 & \\
\hline 5682 & E3 & 12.28 & 0.373 & -29.61 & 2.70 & 6 & 6.62 & 10.22 & 2894 & 0.000 & 0.707 & 9.8 & 1.0 & \\
\hline 5797 & $\mathrm{E} 67$ & 12.38 & 0.366 & -16.47 & 15.47 & 5 & 34.60 & 50.77 & 1488 & 0.000 & 0.358 & 7.9 & 1.4 & \\
\hline 5920 & E3 & 12.05 & 0.374 & -28.68 & 0.57 & 6 & 1.40 & 1.10 & 2885 & 0.319 & 0.179 & 29.9 & 3.0 & \\
\hline 5930 & E75 & 12.00 & 0.187 & -32.16 & 2.31 & 1 & 2.31 & 1.00 & 0 & - & 0.607 & 23.2 & 5.6 & $\mathrm{pm}$ \\
\hline 6097 & E15 & 13.51 & 0.374 & -30.60 & 0.67 & 4 & 1.33 & 1.57 & 2174 & 0.062 & 0.920 & 10.0 & 1.8 & \\
\hline 6176 & E14 & 13.12 & 0.335 & -31.27 & 1.59 & 3 & 2.75 & 1.02 & 1878 & 0.352 & 0.812 & 34.1 & 5.9 & \\
\hline 6277 & E66 & 12.70 & 0.348 & -30.28 & 0.58 & 5 & 1.29 & 1.37 & 2541 & 0.116 & 0.757 & 17.5 & 2.1 & \\
\hline 6282 & A 1225 & 14.11 & 0.394 & -31.28 & 0.39 & 3 & 0.65 & 0.97 & 1397 & 0.405 & 0.727 & 6.4 & 4.3 & \\
\hline 6333 & E6 & 10.38 & 0.779 & -30.89 & 0.08 & 11 & 0.26 & 0.78 & 5156 & 0.800 & 0.868 & 0.0 & 0.0 & $\mathrm{M}^{*}$ \\
\hline 6342 & E37 & 12.22 & 0.360 & -35.69 & 2.81 & 3 & 4.87 & 1.76 & 1489 & 0.046 & 0.118 & $>50$ & - & \\
\hline 6375 & E74 & 12.12 & 0.388 & -30.77 & 0.84 & 6 & 2.07 & 1.60 & 2211 & 0.027 & 0.995 & 32.1 & 3.2 & \\
\hline 6686 & E65 & 10.85 & 0.728 & -30.77 & 0.07 & 21 & 3.51 & 9.91 & 5155 & 0.000 & 0.990 & 1.1 & 0.0 & $\mathrm{MO}$ \\
\hline 6725 & A1227 & 13.25 & 0.384 & -33.11 & 18.82 & 2 & 26.61 & 19.39 & 774 & 0.000 & 0.901 & 16.7 & 2.8 & \\
\hline 6726 & A1230 & 13.23 & 0.386 & -30.00 & 0.53 & 4 & 36.70 & 37.69 & 1117 & 0.000 & 0.620 & 6.7 & 3.8 & $\mathrm{sb} 2 *$ \\
\hline 6733 & E35 & 12.50 & 0.337 & -29.68 & 6.23 & 5 & 12.33 & 2.05 & 1890 & 0.000 & 0.866 & 14.7 & 4.7 & $\mathrm{sb} 2 *$ \\
\hline 6752 & $\mathrm{E}$ & 13.72 & 0.368 & -32.15 & 1.37 & 3 & 2.37 & 3.34 & 1104 & 0.000 & 0.484 & 7.3 & 2.0 & \\
\hline 6762 & A 1215 & 14.13 & 0.655 & -74.80 & 0.74 & 1 & 0.74 & 1.00 & 0 & - & 0.000 & 10.7 & 5.6 & \\
\hline 6770 & E78 & 13.02 & 0.338 & -22.89 & 4.06 & 1 & 4.06 & 1.00 & 0 & - & 0.068 & 27.8 & 17.2 & $\mathrm{pm}$ \\
\hline 6905 & A1228 & 12.94 & 0.344 & -28.48 & 1.03 & 5 & 2.30 & 2.30 & 2175 & 0.001 & 0.198 & 15.7 & 2.3 & \\
\hline 7016 & E34 & 13.42 & 0.358 & -29.89 & 0.73 & 4 & 1.21 & 0.84 & 2897 & 0.556 & 0.590 & 25.2 & 2.7 & \\
\hline 7036 & E73 & 14.04 & 0.413 & 20.90 & 57.25 & 2 & 80.96 & 86.33 & 346 & 0.000 & 0.367 & 6.1 & 7.0 & \\
\hline 7111 & E17 & 12.62 & 0.341 & -29.37 & 4.09 & 1 & 4.09 & 1.00 & 0 & - & 0.749 & 10.0 & 12.1 & $\mathrm{pm}$ \\
\hline 7181 & A 4222 & 13.04 & 0.352 & -13.94 & 1.01 & 1 & 1.01 & 1.00 & 0 & - & 0.000 & 10.1 & 4.1 & \\
\hline 7254 & E16 & 12.38 & 0.357 & -33.10 & 5.40 & 2 & 7.63 & 3.11 & 1091 & 0.002 & 0.675 & 67.8 & 6.8 & \\
\hline 7309 & E61 & 13.12 & 0.360 & -34.50 & 8.15 & 4 & 16.30 & 11.62 & 2192 & 0.000 & 0.651 & 24.0 & 2.4 & \\
\hline 7492 & E38 & 13.57 & 0.421 & -46.19 & 0.66 & 2 & 0.33 & 0.35 & 1483 & 0.723 & 0.000 & 11.6 & 2.4 & \\
\hline 7646 & E12 & 10.32 & 0.661 & -31.18 & 0.13 & 7 & 0.22 & 0.63 & 4873 & 0.896 & 0.595 & 1.1 & 1.4 & M \\
\hline 7753 & A1211 & 13.97 & 0.417 & -32.18 & 1.67 & 2 & 2.36 & 3.50 & 1491 & 0.001 & 0.520 & 10.1 & 1.8 & \\
\hline 7754 & E99 & 12.38 & 0.347 & -31.74 & 0.55 & 5 & 0.91 & 0.75 & 2886 & 0.699 & 0.525 & 28.1 & 2.8 & \\
\hline 7947 & E62 & 13.48 & 0.832 & 9.47 & 0.88 & 1 & 0.88 & 1.00 & 0 & - & 0.000 & 7.3 & 5.4 & \\
\hline 8080 & E77 & 12.08 & 0.380 & -29.56 & 0.92 & 5 & 2.06 & 2.71 & 1846 & 0.000 & 0.483 & 13.8 & 1.0 & \\
\hline 8263 & E70 & 12.06 & 0.392 & -31.24 & 0.73 & 5 & 1.63 & 1.22 & 1848 & 0.206 & 0.766 & 65: & - & \\
\hline 8276 & E5 & 12.81 & 0.329 & -29.84 & 1.14 & 4 & 2.29 & 1.47 & 2594 & 0.099 & 0.616 & 23.9 & 2.4 & \\
\hline 8302 & A1208 & 12.60 & 0.358 & -30.58 & 3.49 & 4 & 9.35 & 9.53 & 1143 & 0.000 & 0.962 & 9.9 & 5.0 & $\mathrm{sb} 2^{*}$ \\
\hline 8303 & A4106 & 13.71 & 0.375 & -31.09 & 0.66 & 4 & 1.31 & 1.35 & 2181 & 0.141 & 0.835 & 13.6 & 1.6 & \\
\hline 8437 & E79 & 13.55 & 0.360 & -28.94 & 0.65 & 4 & 1.17 & 0.90 & 1510 & 0.502 & 0.249 & 21.8 & 2.3 & \\
\hline 8480 & E39 & 12.31 & 0.373 & -29.59 & 0.73 & 5 & 1.50 & 0.92 & 2545 & 0.504 & 0.469 & 27.6 & 10.7 & \\
\hline 8506 & E63 & 12.60 & 0.345 & -38.66 & 12.40 & 3 & 21.47 & 13.06 & 406 & 0.000 & 0.527 & 22.2 & 3.9 & \\
\hline 8540 & E98 & 10.87 & 0.694 & -30.36 & 0.14 & 7 & 0.30 & 0.83 & 4872 & 0.673 & 0.614 & 1.0 & 0.0 & M \\
\hline 8642 & E33 & 13.57 & 0.379 & -29.67 & 1.26 & 4 & 2.52 & 3.11 & 2898 & 0.000 & 0.569 & 11.6 & 1.4 & \\
\hline 8665 & L244 & 11.11 & 0.526 & -30.53 & 0.16 & 27 & 10.92 & 18.63 & 4472 & 0.000 & 0.773 & 1.6 & 1.5 & $\mathrm{MO}, \mathrm{sb} 2$ \\
\hline 8803 & E64 & 13.72 & 0.370 & -29.91 & 0.40 & 4 & 0.51 & 0.64 & 1797 & 0.747 & 0.570 & 9.2 & 1.7 & \\
\hline 8878 & A1114 & 13.65 & 0.406 & 6.39 & 2.03 & 2 & 2.88 & 3.07 & 1107 & 0.002 & 0.000 & 7.2 & 3.6 & \\
\hline 9025 & E60 & 10.87 & 0.673 & -30.46 & 0.14 & 8 & 0.25 & 0.65 & 4872 & 0.901 & 0.705 & 1.9 & 1.5 & M \\
\hline 9054 & A1108 & 14.05 & 0.395 & -31.76 & 0.81 & 3 & 1.41 & 2.23 & 1404 & 0.007 & 0.545 & 7.0 & 2.6 & \\
\hline 9115 & E71 & 12.92 & 0.353 & -31.02 & 0.42 & 5 & 0.94 & 1.12 & 2896 & 0.305 & 0.862 & 13.5 & 1.2 & \\
\hline 9122 & E58 & 10.87 & 0.717 & -30.58 & 0.13 & 8 & 0.30 & 0.83 & 4872 & 0.703 & 0.920 & 0.0 & 0.0 & M \\
\hline 9263 & A4107 & 13.91 & 0.385 & -29.54 & 1.22 & 3 & 2.11 & 2.84 & 1127 & 0.000 & 0.518 & 7.7 & 3.4 & \\
\hline 9266 & E97 & 11.62 & 0.772 & 19.33 & 0.49 & 1 & 0.49 & 1.00 & 0 & - & 0.000 & 5.8 & 2.6 & \\
\hline 9278 & E59 & 12.87 & 0.354 & -28.35 & 1.75 & 5 & 20.81 & 21.59 & 1499 & 0.000 & 0.288 & 4.2 & 5.2 & $\mathrm{sb} 2 *$ \\
\hline 9357 & E18 & 11.77 & 0.381 & -31.51 & 1.16 & 6 & 2.85 & 4.04 & 2898 & 0.000 & 0.685 & 16.3 & 1.0 & \\
\hline
\end{tabular}


Table 1. continued.

\begin{tabular}{|c|c|c|c|c|c|c|c|c|c|c|c|c|c|c|}
\hline MEI & Other & $V$ & $b-y$ & $<R V>$ & $\sigma_{<V>}$ & $n$ & $\sigma_{1}$ & $\mathrm{O} / \mathrm{C}$ & $\Delta T$ & $P\left(\chi^{2}\right)$ & $P(R V)$ & $v \sin i$ & $\sigma_{v}$ & Rem \\
\hline 9488 & A1104 & 13.53 & 0.640 & 42.59 & 0.59 & 1 & 0.59 & 1.00 & 0 & - & 0.000 & 2.2 & 0.0 & \\
\hline 9532 & E7 & 14.17 & 0.402 & -30.51 & 0.38 & 4 & 0.76 & 1.33 & 1405 & 0.158 & 0.867 & 2.1 & 3.0 & \\
\hline 9536 & E56 & 12.18 & 0.378 & -28.28 & 0.71 & 5 & 0.73 & 0.46 & 2545 & 0.937 & 0.122 & 27.8 & 10.8 & \\
\hline 9640 & E32 & 13.13 & 0.341 & -22.30 & 6.04 & 4 & 12.09 & 14.17 & 1845 & 0.000 & 0.173 & 14.9 & 1.5 & \\
\hline 9745 & E53 & 13.44 & 0.403 & -31.07 & 0.24 & 7 & 0.28 & 0.44 & 2900 & 0.982 & 0.832 & 8.3 & 1.2 & \\
\hline 9791 & E96 & 10.34 & 0.788 & -31.44 & 0.12 & 8 & 0.33 & 0.95 & 4872 & 0.515 & 0.389 & 0.1 & 0.0 & $\mathrm{M}$ \\
\hline 9826 & E54 & 12.13 & 0.443 & 9.30 & 0.47 & 4 & 0.94 & 1.66 & 1844 & 0.042 & 0.000 & 3.1 & 2.1 & \\
\hline 10019 & A2202 & 12.68 & 0.375 & -29.15 & 1.25 & 4 & 2.49 & 2.40 & 1494 & 0.000 & 0.398 & 23.8 & 4.5 & sb3* \\
\hline 10029 & E55 & 12.23 & 0.389 & -31.60 & 11.08 & 4 & 16.25 & 14.78 & 2211 & 0.000 & 0.940 & 14.8 & 10.9 & $\mathrm{sb} 2 *$ \\
\hline 10195 & L241 & 10.86 & 0.720 & -30.74 & 0.10 & 22 & 8.15 & 19.91 & 4098 & 0.000 & 0.980 & 4.8 & 0.5 & $\mathrm{MO}$ \\
\hline 10387 & E0 & 13.57 & 0.774 & -34.22 & 1.64 & 1 & 1.64 & 1.00 & 0 & - & 0.057 & 5.2 & 9.8 & $\mathrm{pm}$ \\
\hline 10393 & E8 & 10.67 & 0.666 & -31.54 & 0.14 & 8 & 0.37 & 0.96 & 4873 & 0.529 & 0.325 & 0.1 & 1.8 & $\mathrm{M}$ \\
\hline 10807 & A 2105 & 13.95 & 0.429 & -31.38 & 0.86 & 4 & 1.72 & 1.89 & 2181 & 0.015 & 0.712 & 11.3 & 2.0 & \\
\hline 10811 & E81 & 13.31 & 0.352 & -33.45 & 5.08 & 3 & 8.80 & 5.29 & 1923 & 0.000 & 0.610 & 27.8 & 4.4 & \\
\hline 10866 & E28 & 12.19 & 0.388 & -30.94 & 1.61 & 7 & 4.25 & 2.06 & 2248 & 0.000 & 0.934 & 36.2 & 3.6 & \\
\hline 10963 & E95 & 11.95 & 0.514 & -29.93 & 0.30 & 6 & 0.74 & 1.21 & 2887 & 0.206 & 0.573 & 12.0 & 0.9 & \\
\hline 11020 & A3205 & 14.26 & 0.422 & -11.23 & 0.65 & 1 & 0.65 & 1.00 & 0 & - & 0.000 & 2.9 & 5.4 & \\
\hline 11123 & E82 & 13.73 & 0.392 & -43.00 & 0.98 & 1 & 0.98 & 1.00 & 0 & - & 0.000 & 15.4 & 4.3 & \\
\hline 11218 & M812 & 11.06 & 0.676 & -30.36 & 0.37 & 4 & 0.73 & 2.09 & 1815 & 0.004 & 0.643 & 0.5 & 1.8 & $\mathrm{M}^{*}$ \\
\hline 11453 & E27 & 10.16 & 0.652 & -29.41 & 0.19 & 7 & 0.51 & 1.39 & 4872 & 0.083 & 0.093 & 2.1 & 1.3 & $\mathrm{M}$ \\
\hline 11504 & E94 & 11.63 & 0.394 & -28.02 & 0.61 & 6 & 1.49 & 2.37 & 1489 & 0.000 & 0.080 & 10.3 & 1.8 & $\mathrm{sb} 3^{*}$ \\
\hline 11507 & E11 & 12.41 & 0.309 & -22.64 & 6.56 & 3 & 11.37 & 2.78 & 2208 & 0.001 & 0.227 & 20.2 & 7.9 & \\
\hline 11662 & $\mathrm{~A} 2217$ & 14.01 & 0.461 & -28.27 & 0.81 & 1 & 0.81 & 1.00 & 0 & - & 0.132 & 13.1 & 2.9 & $\mathrm{pm}$ \\
\hline 11670 & E41 & 13.14 & 0.369 & -34.71 & 5.20 & 4 & 10.39 & 12.28 & 1842 & 0.000 & 0.464 & 11.2 & 1.6 & \\
\hline 11709 & E19 & 12.24 & 0.412 & -31.41 & 0.90 & 5 & 2.01 & 1.58 & 1841 & 0.041 & 0.702 & 32.1 & 3.2 & \\
\hline 11808 & E50 & 12.41 & 0.362 & -32.65 & 4.09 & 4 & 8.18 & 9.53 & 1495 & 0.000 & 0.663 & 13.5 & 1.4 & \\
\hline 11887 & E10 & 11.53 & 0.373 & -28.95 & 2.77 & 4 & 5.53 & 2.46 & 1845 & 0.001 & 0.562 & 31.3 & 7.5 & \\
\hline 12008 & E49 & 13.38 & 0.365 & -26.06 & 2.59 & 4 & 5.18 & 4.95 & 2897 & 0.000 & 0.113 & 14.4 & 3.6 & \\
\hline 12137 & A3213 & 14.08 & 0.455 & -31.00 & 0.46 & 2 & 0.65 & 1.05 & 714 & 0.306 & 0.874 & 3.1 & 6.6 & \\
\hline 12227 & E93 & 8.80 & 1.031 & -29.80 & 0.14 & 7 & 0.37 & 1.16 & 4872 & 0.243 & 0.226 & 1.9 & 1.1 & M \\
\hline 12270 & E26 & 12.99 & 0.355 & -30.97 & 0.40 & 6 & 0.99 & 1.03 & 2893 & 0.385 & 0.888 & 16.8 & 1.7 & \\
\hline 12362 & E48 & 12.77 & 0.749 & -77.60 & 1.82 & 2 & 2.57 & 4.85 & 1830 & 0.000 & 0.000 & 4.0 & 0.0 & \\
\hline 12453 & A2214 & 13.19 & 0.346 & -28.56 & 2.84 & 3 & 4.91 & 7.02 & 1107 & 0.000 & 0.490 & 6.8 & 2.0 & \\
\hline 12854 & E86 & 13.74 & 0.383 & -29.49 & 0.71 & 5 & 1.60 & 1.82 & 2544 & 0.014 & 0.429 & 14.0 & 1.4 & \\
\hline 12889 & A3226 & 14.12 & 0.423 & -33.29 & 0.46 & 2 & 0.18 & 0.27 & 714 & 0.784 & 0.094 & 5.9 & 2.7 & \\
\hline 12935 & E22 & 10.92 & 0.681 & -29.87 & 0.13 & 8 & 0.28 & 0.79 & 4873 & 0.764 & 0.260 & 1.6 & 0.0 & $\mathrm{M}$ \\
\hline 13113 & $\mathrm{E} 45$ & 14.18 & 0.430 & -29.63 & 0.34 & 3 & 0.55 & 0.92 & 1405 & 0.431 & 0.445 & 4.2 & 4.3 & \\
\hline 13238 & E44 & 12.65 & 0.352 & -31.70 & 0.94 & 6 & 2.30 & 3.08 & 1845 & 0.000 & 0.585 & 9.1 & 1.8 & \\
\hline 13247 & $\mathrm{E} 87$ & 13.80 & 0.699 & -14.46 & 0.82 & 1 & 0.82 & 1.00 & 0 & - & 0.000 & 7.6 & 5.7 & \\
\hline 13324 & E85 & 13.65 & 0.388 & -28.34 & 0.45 & 4 & 0.19 & 0.21 & 2213 & 0.988 & 0.109 & 16.2 & 1.3 & \\
\hline 13482 & E88 & 14.40 & 0.438 & -28.31 & 2.53 & 2 & 3.57 & 4.00 & 1523 & 0.000 & 0.400 & 3.6 & 8.9 & \\
\hline 13755 & $\mathrm{E} 25$ & 12.66 & 0.366 & -32.15 & 0.42 & 4 & 0.67 & 0.80 & 1841 & 0.590 & 0.354 & 9.1 & 1.8 & \\
\hline 14290 & E91 & 10.58 & 0.652 & -31.37 & 0.07 & 27 & 9.27 & 23.90 & 4470 & 0.000 & 0.436 & 0.2 & 1.0 & $\mathrm{MO}$ \\
\hline 14331 & A2230 & 13.76 & 0.408 & -33.57 & 0.72 & 2 & 1.01 & 1.04 & 347 & 0.299 & 0.081 & 10.6 & 2.6 & \\
\hline 14364 & E24 & 12.76 & 0.368 & -31.34 & 7.64 & 4 & 15.29 & 18.51 & 1841 & 0.000 & 0.941 & 12.4 & 1.8 & \\
\hline 14527 & E83 & 10.90 & 0.688 & -31.85 & 0.26 & 7 & 0.68 & 1.83 & 4873 & 0.004 & 0.185 & 2.2 & 1.4 & $\mathrm{M}^{*}$ \\
\hline 14560 & E84 & 13.09 & 0.384 & -33.45 & 0.39 & 4 & 0.56 & 0.71 & 2209 & 0.689 & 0.071 & 15.3 & 1.4 & \\
\hline 14641 & E23 & 10.65 & 0.684 & -31.19 & 0.07 & 21 & 1.86 & 5.17 & 5155 & 0.000 & 0.583 & 0.9 & 0.0 & $\mathrm{MO}$ \\
\hline- & M811 & 10.72 & - & -31.13 & 0.21 & 4 & 0.42 & 1.25 & 1815 & 0.200 & 0.647 & 0.4 & 1.7 & $\mathrm{M}$ \\
\hline- & M817 & 10.68 & - & -31.75 & 0.21 & 4 & 0.42 & 1.20 & 1815 & 0.230 & 0.220 & 1.6 & 0.0 & M \\
\hline
\end{tabular}

Of the 104 observed stars, 45 (43\%) are found to be binaries, but the fractions are very different for cluster and field stars $(42 / 86$ or $49 \%$, vs. $3 / 13$ or $23 \%)$. This is readily understandable because field stars were not monitored systematically once their non-member status had become clear. In contrast, the lower binary frequency among the cluster giants ( $7 / 19$ or $37 \%)$ than among the main-sequence stars (35/67 or $52 \%)$ is probably real, as the red giants have been monitored for longer periods and with better precision than the hotter, faster-rotating 
Table 2. Revised orbital elements, with mean errors, for the red giant binaries in IC 4651. After the MEI numbers, Lindoff (1972) numbers (L) are also given to facilitate comparison with Mermilliod et al. (1995).

\begin{tabular}{|c|c|c|c|c|c|}
\hline Orbital element: & 6686 (L97) & 8665 (L244) & 10195 (L241) & 14641 (L236) & 14290 (L139) \\
\hline \multirow[t]{2}{*}{$P[\mathrm{~d}]$} & 5021 & 1318.2 & 75.162 & 335.540 & 2764 \\
\hline & 40 & 1.5 & 0.012 & 0.057 & 30 \\
\hline \multirow[t]{2}{*}{$T$ [HJD-2440000] } & 5798 & 7860.6 & 9031.5 & 8956.76 & 8133 \\
\hline & 89 & 2.5 & 1.7 & 0.36 & 79 \\
\hline \multirow[t]{2}{*}{$e$} & 0.18 & 0.773 & 0.090 & 0.000 & 0.19 \\
\hline & 0.02 & 0.017 & 0.016 & 0.007 & 0.04 \\
\hline \multirow[t]{2}{*}{$\omega\left[^{\circ}\right]$} & 111.3 & 359.6 & 57.4 & 270.0 & 58.8 \\
\hline & 7.2 & 5.0 & 7.7 & - & 10.8 \\
\hline \multirow[t]{2}{*}{$\gamma\left[\mathrm{km} \mathrm{s}^{-1}\right]$} & -30.78 & -30.53 & -30.74 & -31.37 & -31.19 \\
\hline & 0.07 & .16 & 0.10 & 0.05 & 0.07 \\
\hline \multirow[t]{2}{*}{$K_{1}\left[\mathrm{~km} \mathrm{~s}^{-1}\right]$} & 4.95 & 20.1 & 11.04 & 15.96 & 2.62 \\
\hline & 0.11 & 1.0 & 0.14 & 0.07 & 0.10 \\
\hline \multirow[t]{2}{*}{$K_{2}\left[\mathrm{~km} \mathrm{~s}^{-1}\right]$} & & 17.4 & & & \\
\hline & & 1.3 & & & \\
\hline \multirow[t]{2}{*}{$a_{1} \sin i[\mathrm{Gm}]$} & 336.1 & 231 & 11.37 & 73.66 & 97.6 \\
\hline & 7.9 & 14 & 0.15 & 0.34 & 4.0 \\
\hline \multirow[t]{2}{*}{$a_{2} \sin i[\mathrm{Gm}]$} & & 200 & & & \\
\hline & & 16 & & & \\
\hline \multirow[t]{2}{*}{$\mathrm{f}(m)\left[M_{\odot}\right]$} & 0.0600 & & 0.0104 & 0.1415 & 0.0049 \\
\hline & 0.0043 & & 0.0004 & 0.0019 & 0.0006 \\
\hline \multirow[t]{2}{*}{$m_{1} \sin ^{3} i\left[M_{\odot}\right]$} & & 0.85 & & & \\
\hline & & 0.10 & & & \\
\hline \multirow[t]{2}{*}{$m_{2} \sin ^{3} i\left[M_{\odot}\right]$} & & 0.98 & & & \\
\hline & & 0.11 & & & \\
\hline$\sigma\left[\mathrm{km} \mathrm{s}^{-1}\right]$ & 0.33 & 0.67 & 0.45 & 0.23 & 0.30 \\
\hline$n_{\text {obs }}$ & 21 & 22 & 28 & 27 & 21 \\
\hline
\end{tabular}

F-type main-sequence stars. Destruction of the closest systems after Roche lobe overflow and mass exchange as the primaries expand upon leaving the main sequence provides a natural explanation of this difference.

\subsection{Cluster membership}

The deviation of the individual stellar radial velocities from the cluster mean, compared to the combined effects of individual measuring uncertainties $\sigma_{<V>}$ and the internal velocity dispersion in the cluster, allows to compute individual membership probabilities $P(R V)$ as discussed by Nordström et al. (1997). The conservative limit $P(R V)<0.01$ is taken to identify the definite nonmembers. Stars with a $P(R V)$ indicating membership, but based only on a single radial velocity (hence unknown binary status), are labeled "probable" members, provided their position in the CMD is also consistent with membership (see Table 1).

First, we must compute the cluster mean velocity. The 12 non-variable red giants in Table 1 yield a mean velocity for IC 4651 of $-30.70 \pm 0.22 \mathrm{~km} \mathrm{~s}^{-1}$, similar to that reported by Mermilliod et al. (1995) from all 19 cluster giants $\left(-31.0 \pm 0.2 \mathrm{~km} \mathrm{~s}^{-1}\right)$. The standard deviation from the cluster average of a single mean velocity is $0.75 \mathrm{~km} \mathrm{~s}^{-1}$ for the red giants; correcting for the average observational error $\left(0.15 \mathrm{~km} \mathrm{~s}^{-1}\right)$, we find a true cluster velocity dispersion of $0.74 \mathrm{~km} \mathrm{~s}^{-1}$. The velocity dispersion of IC 4651 is thus much larger than that of NGC 3680 $\left(0.30 \mathrm{kms}^{-1}\right)$, reflecting a larger mass, but maybe also indicating a difference in the dynamical evolution of the two clusters.

Using these values for the cluster mean velocity and internal dispersion in IC 4651 we computed membership probabilities $P(R V)$ for all the stars in Table 1 . The mean radial velocity of the 32 single F-type main-sequence and turnoff members so defined is $-30.82 \pm 0.28 \mathrm{~km} \mathrm{~s}^{-1}$, with a standard deviation per star of $1.59 \mathrm{~km} \mathrm{~s}^{-1}$, or $1.42 \mathrm{~km} \mathrm{~s}^{-1}$ once the average measuring uncertainty of $0.72 \mathrm{~km} \mathrm{~s}^{-1}$ has been subtracted. The difference from the mean velocity of the giants, only $0.12 \mathrm{~km} \mathrm{~s}^{-1}$, is much smaller than that in NGC 3680 (Nordström et al. 1997), probably because of the improved zero-point calibration used in the present study (cf. Fig. 2b). Within the uncertainties, it remains compatible with the difference in gravitational red-shift between dwarfs and giants, but does not require the use of different mean velocities for the two groups of stars. The final membership probabilities $P(R V)$, listed in Table 1, have therefore been computed with the final weighted mean velocity: $-30.76 \pm 0.20 \mathrm{~km} \mathrm{~s}^{-1}$.

With these definitions, the $P(R V)$ values in Table 1 classify 86 stars as certain and 5 stars as probable cluster members, while only 13 of the 104 observed stars are found to be field stars, a much lower percentage than in 

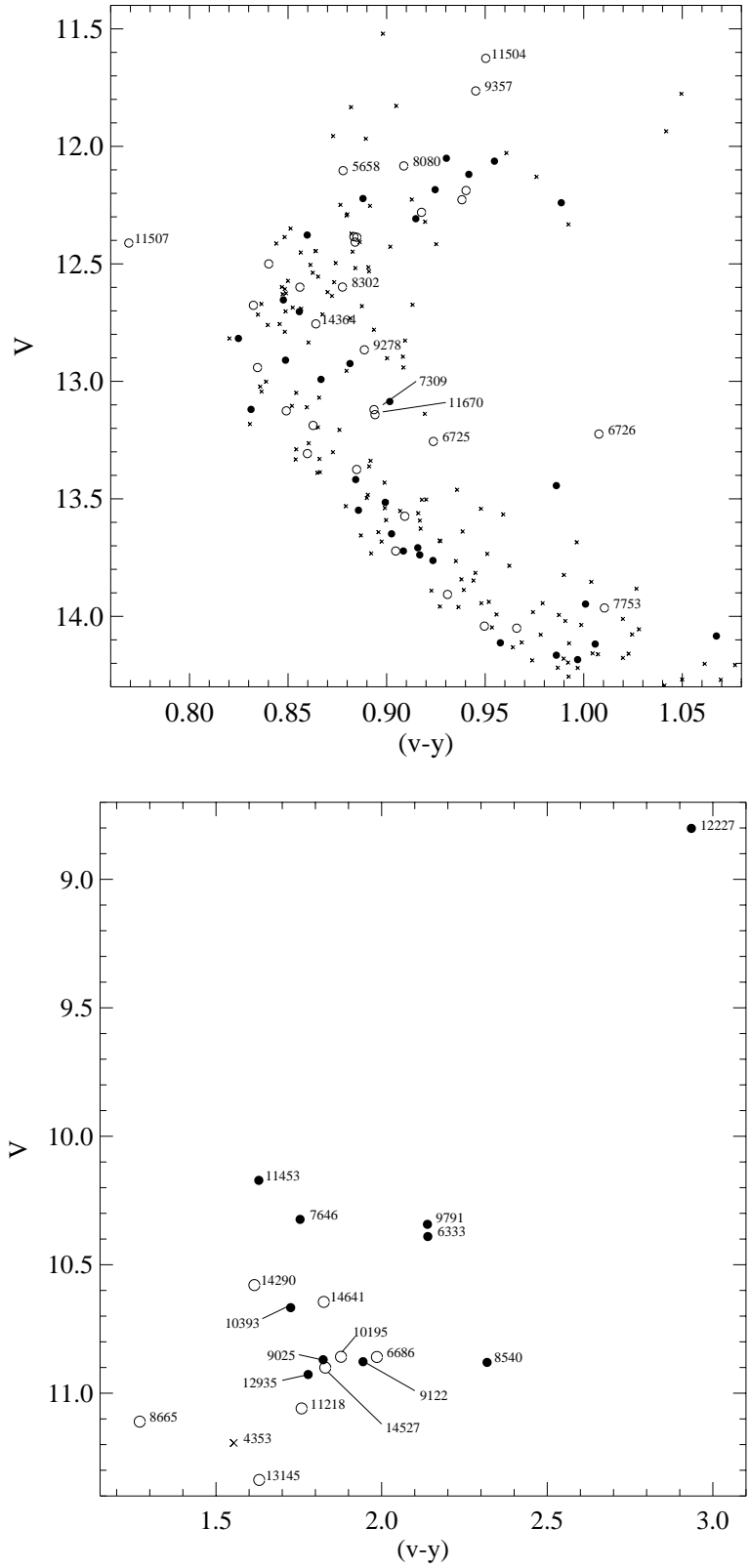

Fig. 3. a) The upper main sequence and turn-off of IC 4651. Single and binary cluster members are shown as filled and open circles, respectively; crosses denote stars without radialvelocity data. MEI numbers are given for the 13 binary stars more than $\sim 0.1^{\mathrm{m}}$ above the best-fit isochrone. b) The red giant region (symbols as a)); MEI numbers are given for all stars.

NGC 3680. Of the 13 field stars, 2 are single, 3 are binaries, and 8 are of unknown type. Figure 3 shows the turn-off and red giant region of the CMD of IC 4651, identifying the different categories of stars. Because the new photometry by Meibom (2000) approximately doubled the number of cluster stars just as the CORAVEL observing campaign came to an end, the CMD still contains many stars without cluster membership and duplicity data; these are ripe targets for follow-up with multi-fiber spectrographs.

\subsection{Reddening, metallicity, and distance of IC 4651}

Several determinations of reddening and metal abundance of IC 4651 have been made; see Kjeldsen \& Frandsen (1991) for earlier results. Most recently, Anthony-Twarog \& Twarog (2000) made determinations of the reddening and metallicity of IC 4651: $E_{(b-y)}=0.062 \pm 0.003$ (sem), $[\mathrm{Fe} / \mathrm{H}]=0.077 \pm 0.012$ (based on the intrinsic colour relation of Olsen (1984)), and $E_{(b-y)}=0.071,[\mathrm{Fe} / \mathrm{H}]=0.115$ (based on the intrinsic colour relation of Nissen 1988).

On the basis of uvby- $\mathrm{H}_{\beta}$ photoelectric and CCD photometry Nissen (1988) reported $[\mathrm{Fe} / \mathrm{H}]=0.18 \pm 0.05$ and $E_{(b-y)}=0.076 \pm 0.012$ as a mean of 10 individual measurements. Anthony-Twarog \& Twarog (1987) reported $[\mathrm{Fe} / \mathrm{H}]=0.23 \pm 0.02$ and $E_{(b-y)}=0.064 \pm 0.006$ as a mean of 66 and 11 measurements, respectively. The new radial velocity data reveal that of the 10 stars used by Nissen (1988), only 2 are single members, 7 are binary members and one is of unknown type and membership. Of the 11 stars used by Anthony-Twarog \& Twarog (1987) for determination of reddening, 4 are single members, 5 are binary members, and 2 are non-members.

Radial velocity data exist for 46 of the 66 stars used by Anthony-Twarog \& Twarog (1987) to determine [Fe/H]. 16 of those are single members. Using only single member stars, $[\mathrm{Fe} / \mathrm{H}]=0.16$ and $E_{(b-y)}=0.078$ are derived as mean values from the study of Nissen (1988), and $[\mathrm{Fe} / \mathrm{H}]=$ $0.20, E_{(b-y)}=0.074$ from the study of Anthony-Twarog \& Twarog (1987), making the two studies more consistent. Adopting a mean reddening of $E_{(b-y)}=0.076 \pm 0.012$ (equivalent to $\left.E_{(B-V)}=0.11\right)$, good agreement with the result by Eggen (1971) $\left(E_{(B-V)}=0.13 \pm 0.02\right)$ is obtained. Comparison of the metallicity index $\left(m_{1}\right)$ between the present study and Nissen (1988) shows a mean difference $\left(m_{1_{\mathrm{MEI}}}-m_{1_{\mathrm{N} 88}}\right)$ of $\sim-0.005$. Thus, the mean metallicity determined for single member stars from the studies of Nissen (1988) and Anthony-Twarog \& Twarog $(1987)([\mathrm{Fe} / \mathrm{H}]=0.18 \pm 0.05)$, should be reduced to $[\mathrm{Fe} / \mathrm{H}] \sim 0.13 \pm 0.05$.

Very recently, Bragaglia et al. (2001) quote a preliminary determination of $[\mathrm{Fe} / \mathrm{H}]=+0.16 \pm 0.01$ from highresolution spectroscopy of five unspecified clump giants. Within the likely systematic differences between the techniques, this is quite consistent with the photometric value, in contrast to the case of NGC 3680, where Pasquini et al. (2001) found $[\mathrm{Fe} / \mathrm{H}]=-0.17$ from spectroscopy of red giants, while the photometric result of Nissen (1988) and Nordström et al. (1997) was $[\mathrm{Fe} / \mathrm{H}]=+0.1$.

As our final result for the reddening is identical to that adopted in Paper I, the distance to IC 4651 determined there by direct fitting of the Hyades main sequence to that of IC 4651, $\left(m-M_{0}\right)=10.03$ or a distance from the Sun of $1.01 \pm 0.05 \mathrm{kpc}$, is also adopted here.

\section{Stellar evolution in IC 4651}

Although not all candidate members of IC 4651 have radial-velocity data, the identification of member and 
binary stars in the inner region of the cluster (for $V \lesssim$ 14. 5 ), together with the unambiguous identification of the lower main sequence, allow for a critical test of stellar evolution models. Previous discussions, e.g. Nordström et al. (1997) or Anthony-Twarog \& Twarog (2000) and references in these papers, have proved conclusively that convective core overshooting must be included in stellar models for the mass range of IC 4651. However, presentday models differ in their physical description of the phenomenon loosely referred to as "overshooting", leading to subtle differences in model predictions which can only be tested by comparing with high-quality photometric data for established, single cluster members.

\subsection{The models}

Two sets of stellar isochrones are considered here: First, the models by the Geneva group (Schaller et al. 1992), using OPAL radiative opacities (Iglesias et al. 1992a) and an overshooting distance $d$ of $d / H_{\mathrm{p}}=0.20$ in units of the pressure scale height at the classical core boundary. These models were calculated for solar metallicity $(Z=$ $0.02)$. Linear interpolation in the $\log \left(T_{\text {eff }}\right)$ and $M_{\mathrm{V}}$ of the Geneva models for $Z=0.02$ and $Z=0.04$ (Schaller et al. 1992; Schaerer et al. 1993) allows an approximation to models for the observed metallicity of IC 4651.

Second, we also used the new Yale 2000 isochrones (Yi et al. 2002), using improved opacities (Iglesias \& Rogers 1992b) and equations of state (Rogers et al. 1996). Table 1 in $\mathrm{Yi}$ et al. summarizes the input physics. Helium diffusion and convective core overshoot $\left(d / H_{\mathrm{p}}=0.2\right)$ have been taken into consideration. The models are based on a scaled solar chemical composition, and linear interpolation of models for $Z=0.020$ and $Z=0.040$ was used to approximate the metallicity of IC 4651.

\subsection{Colour calibrations}

Colour transformations from effective temperatures to $B-V$ are provided in the Yale models, using both the latest tables of Lejeune et al. (1998) and the modified Green et al. (1987) tables. We have, however, preferred to recompute $(b-y)$ and $(v-y)$ colours from the effective temperatures and metallicities of the models, using the transformation by Edvardsson et al. (1993) for F dwarfs within its range of validity (see Nordström et al. 1997 for a discussion of temperature-colour relations).

\subsection{Isochrone fitting and the age of IC 4651}

Figure 4 shows the CMD of IC 4651 with both Geneva (dot-dashed line) and a Yale 2000 isochrone (solid lines) fits to the cluster sequence. Both models fit the turn-off and upper main-sequence region very well, while the Yale model fits the cluster all the way from the turn-off to the faintest part of the lower main-sequence. Below we will focus on the turn-off region and use our membership

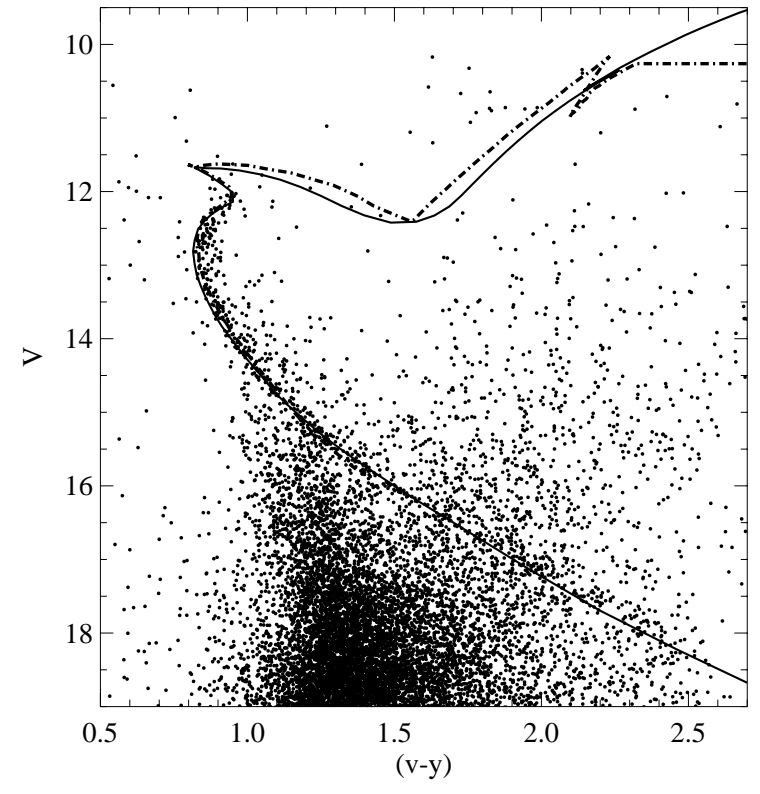

Fig. 4. Yale 20001.8 Gyr (solid; $[\mathrm{Fe} / \mathrm{H}]=0.115$ ) and Geneva $1.58 \mathrm{Gyr}$ (dot-dashed line; $[\mathrm{Fe} / \mathrm{H}]=0.12$ ) isochrones, fit to the entire cluster sequence from the turn-off to the faintest end of the main sequence. The isochrones are shifted in colour and magnitude corresponding to a reddening of $1.68 \times E_{(b-y)}=$ 0.119 . The applied distance moduli are 9.83 and 9.75 , respectively, for the two different models.

and duplicity information to make detailed fits to single member stars. However, the excellent match to the welldefined lower main sequence of IC 4651 not only makes the isochrone fit much more secure; it is also crucial for determining the contribution of the lower main-sequence stars to the cluster mass (Sect. 5). Determining the present-day mass function of IC 4651 is of key importance in the analysis of its dynamical evolution, the main respect in which IC 4651 differs from its "sister" cluster NGC 3680.

The true shape of the cluster turn-off is of critical importance for tests of overshooting in stellar evolution models, and for determining the cluster age. Our radial velocity data have identified 32 single member stars defining the cluster sequence in the turn-off region of the CMD, but in contrast to NGC 3680, the whole lower main sequence is now also well defined in IC 4651. We will fit the two sets of stellar evolution models to the turnoff as defined by the single cluster stars only. Because the theoretical $(b-y)$ colours are only properly calibrated in the F-dwarf domain, the fits are less reliable for $(b-y)_{0} \gtrsim 0.4$ $\left((v-y)_{0} \gtrsim 1.0\right)$.

For clarity, our isochrone fits to IC 4651 are shown in three separate $(b-y)$ vs. $V$ diagrams (Figs. 5,6 , and 7 ). Each diagram shows the single member stars plotted as large solid dots. The 3-4 single stars (MEI 9745, 10807, 12137, 14560; asterisks) significantly to the right of the well-defined single member sequence cannot be explained by scatter due to the uncertainty in the photometry. More radial velocity observations or new proper motion data might reveal that they are in fact long period binary stars 


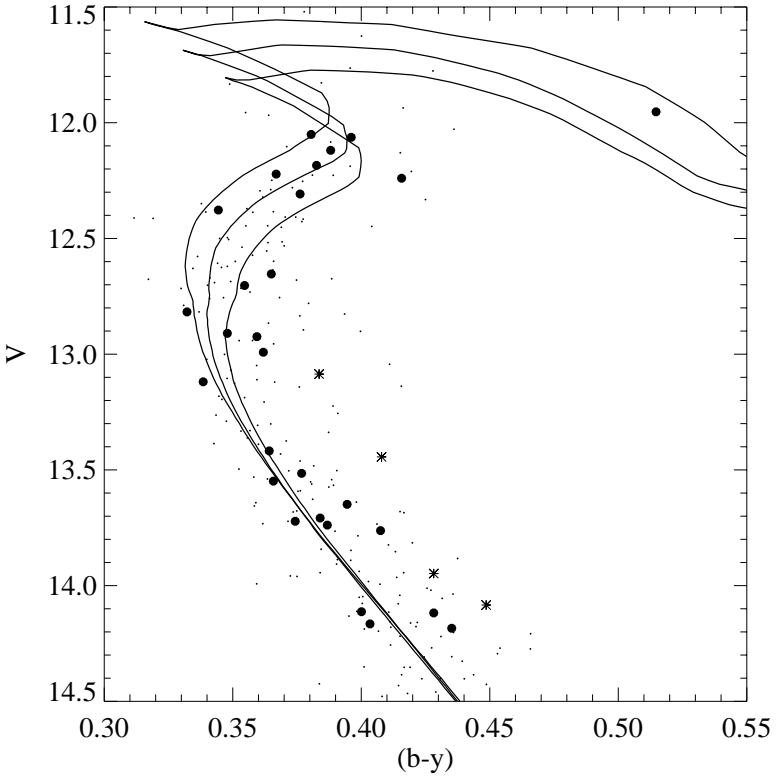

Fig. 5. 1.58, 1.69, and 1.82 Gyr Geneva isochrones for Hyades metallicity fit to the single cluster members (large dots, asterisks) in the turn-off region. Small dots denote binary cluster members and stars without radial-velocity observations. The isochrones are shifted in colour and magnitude corresponding to a reddening of $E_{(b-y)}=0.062$.

and/or non-members with radial velocities indistinguishable from true cluster members. The smaller dots represent binary member stars and photometric cluster members (i.e., stars without $R V$ data).

Figure 5 shows a fit of the 1.58,1.69, and 1.82 Gyr Geneva isochrones, interpolated to the Hyades metallicity $([\mathrm{Fe} / \mathrm{H}] \sim 0.12)$ (see above), fitted for $E_{(b-y)}=0.062$. The isochrones are shifted in magnitude corresponding to $A_{V}=4.3 \times E_{(b-y)}$ (Crawford \& Mandwewala 1976). The applied distance modulus of 9.75 is close to the value (10.03) determined in Meibom (2000) from a direct fit to the Hyades main sequence, and to the values listed in Kjeldsen \& Frandsen (1991) for IC 4651.

Figure 6 shows a fit of the 1.51, 1.58, and 1.69 Gyr Geneva isochrones, interpolated to the Hyades metallicity $([\mathrm{Fe} / \mathrm{H}] \sim 0.12)$, fitted for $E_{(b-y)}=0.071$, and shifted in magnitude corresponding to $A_{V}=4.3 \times E_{(b-y)}$. The applied distance modulus is 9.75 .

The Geneva models fit the unevolved main sequence and the lower part of the turnoff well. They also describe the shape of the redward curvature very precisely all the way to the tip of the main-sequence turn-off. The best fit for $E_{(b-y)}=0.062$ is obtained using the 1.69 Gyr isochrone, while for $E_{(b-y)}=0.071$ the $1.58 \mathrm{Gyr}$ isochrone gives the best fit to the single turn-off members. Note that the $1.58 \mathrm{Gyr}$ Geneva isochrone also fits the only known single sub-giant member. The $\sim 0.1 \mathrm{Gyr}$ age difference between the preferred isochrones for $E_{(b-y)}=0.062$ and $E_{(b-y)}=0.071$ gives a good estimate of the uncertainty introduced by the reddening alone.

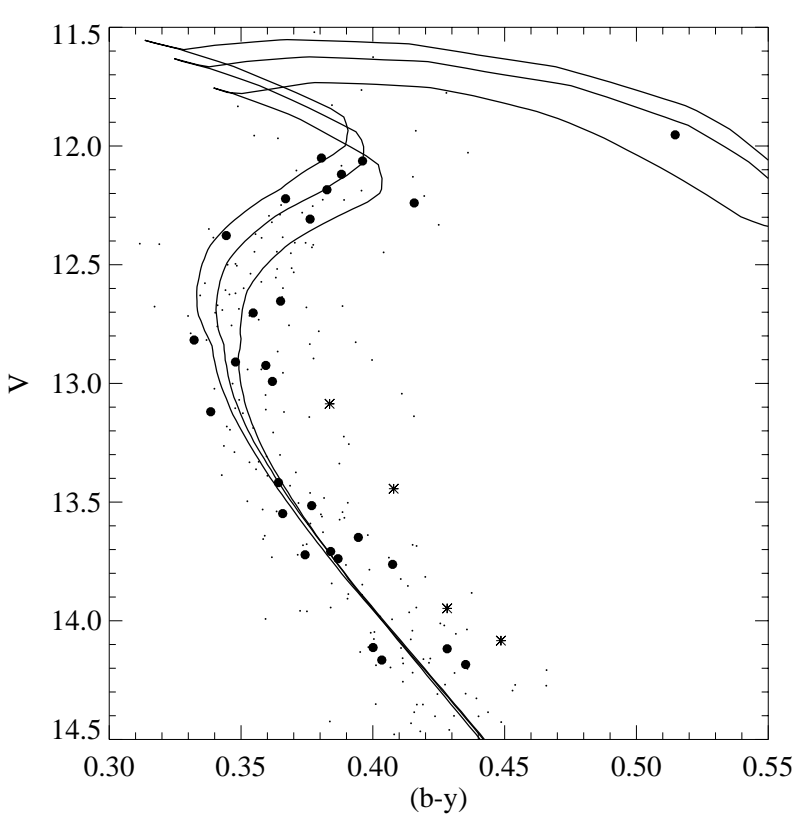

Fig. 6. 1.51, 1.58, and 1.69 Gyr Geneva isochrones for Hyades metallicity fit to the single cluster members in the turn-off region of IC 4651, but for a reddening of $E_{(b-y)}=0.071$. Symbols as in Fig. 5.

Figure 7 shows the Yale 2000 1.7, 1.8 and 1.9 Gyr isochrones ( $\mathrm{Yi}$ et al. 2002) for $[\mathrm{Fe} / \mathrm{H}]=0.115$ and $E_{(b-y)}=0.071$, using a distance modulus of 9.72. These new overshooting models also give a good fit of the turnoff, although the redward curvature is slightly sharper, suggesting a correction (reduction) of the overshooting parameter of the Yale models. Note that the ages of the Yale models are birth-line rather than ZAMS ages, but the difference ( $\lesssim 0.05 \mathrm{Gyr}$ ) is likely to be negligible in all but the very youngest clusters.

Both the 1.58 Gyr Geneva isochrone and the 1.8 Gyr Yale isochrones give excellent fits to the established single cluster members in the turn-off region of IC 4651 for $E_{(b-y)}=0.071$. The relatively small difference of $\sim 0.2 \mathrm{Gyr}$ in age between the best fit of the two different models are due to subtle differences in the stellar model parameters. A similar difference in age between the Geneva models and models constructed using the Yale Stellar Evolution Code is found for NGC 3680; see Nordström et al. (1997) vs. Kozhurina-Platais et al. (1997).

Figure 8 includes giant cluster members on the photometric system of Anthony-Twarog \& Twarog (2000) (see Meibom 2000, Fig. 2) together with the Geneva 1.58 Gyr and Yale $1.8 \mathrm{Gyr}$ isochrones fitted to the single turn-off stars. In the red giant region solid dots represent single stars and open squares represent binary stars. The fact that the isochrones do not fit the single giant members is acceptable because our observations were calibrated primarily for the main-sequence region, and because the uvby colour calibrations are less reliable for red giants.

Previous discussions of stellar models tended to debate simply for or against overshooting, resulting in age estimates for IC 4651 in the whole range from 1.3 Gyr 


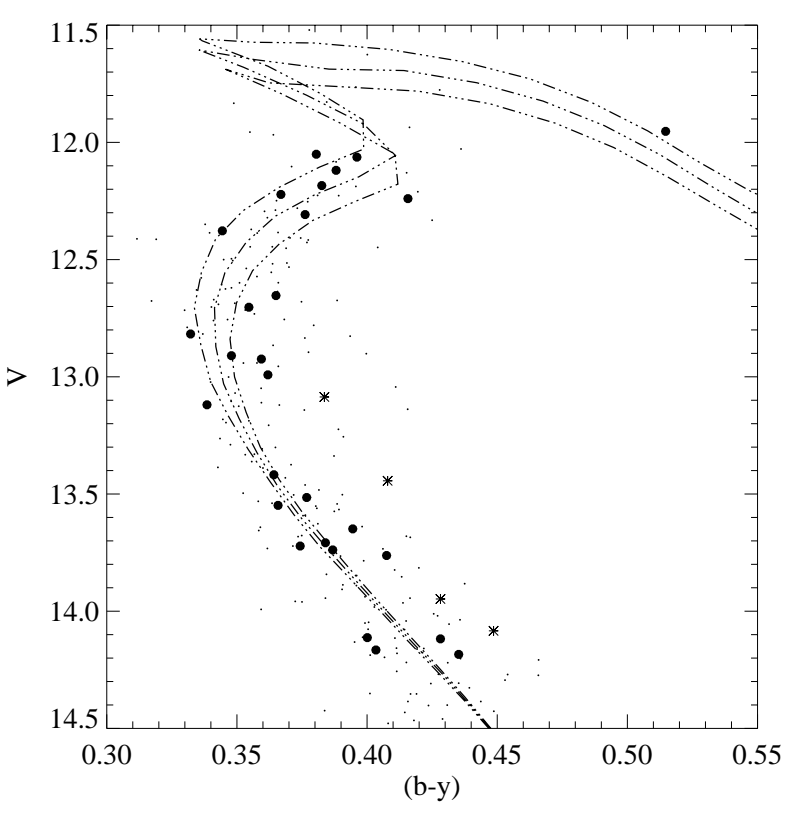

Fig. 7. Yale 2000 isochrones for 1.7, 1.8 and 1.9 Gyr, fitted to the single cluster members in the turn-off region. Symbols as in Figs. 5 and 6 . The isochrones have $[\mathrm{Fe} / \mathrm{H}]=0.115$ and are plotted for $E_{(b-y)}=0.071$ and $(m-M)_{0}=9.72$.

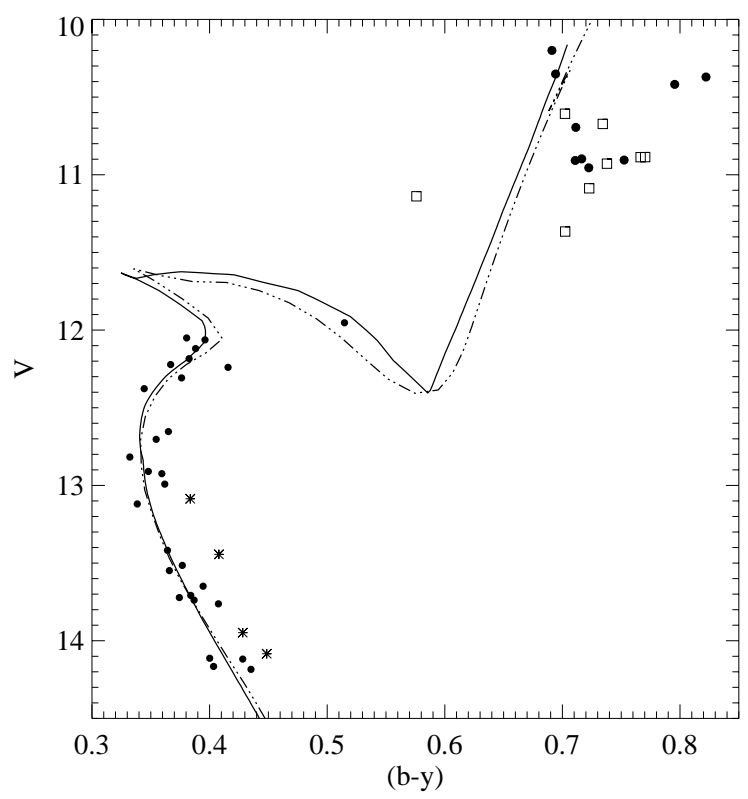

Fig. 8. Turn-off and red giant stars of IC 4651 with the Geneva (1.58 Gyr, solid line) and Yale isochrones (1.8 Gyr, dotted lines) fitted to the single turnoff members. Dots, asterisks: Single cluster stars; open squares: giant binaries.

(Mazzei \& Pigatto 1988) to 4.0 Gyr (Maeder 1990), even when based on the same photometry. The need for extended convective cores in stellar evolution models for stars more massive than the Sun is now well established, but the physical modeling of the overshooting phenomenon needs to be better understood; see, e.g. Giménez et al. (1999) for a comprehensive discussion. The tight sequence of single cluster members defined in IC 4651 by our new data should allow further refinement of the overshooting formalism and lead to improved age estimates for IC 4651 .

Errors in $E_{(b-y)}$ and [Fe/H] will affect the derived cluster age. The uncertainty of the estimated age is derived from the uncertainties in $[\mathrm{Fe} / \mathrm{H}]$ and $E_{(b-y)}$. AnthonyTwarog \& Twarog (2000) give 0.003 and 0.012 as the standard errors of the mean $E_{(b-y)}$ and $[\mathrm{Fe} / \mathrm{H}]$, respectively, based on the colour relations of Olsen (1984), but give no estimate of the uncertainty on the $E_{(b-y)}$ and $[\mathrm{Fe} / \mathrm{H}]$ as based on the colour relations of Nissen (1988). A realistic estimate of the uncertainty in $E_{(b-y)}$ was considered by Nissen (1988) to be \pm 0.012 corresponding to $\sim \pm 0.15 \mathrm{Gyr}$ in the models used here. Combining this with the effect of an uncertainty in $[\mathrm{Fe} / \mathrm{H}]$ of 0.05 dex leads to an estimated total uncertainty on the age of IC 4651 of $\simeq 0.15$ Gyr.

The best fit of both the Geneva and Yale 2000 isochrones is obtained using Hyades metallicity $([\mathrm{Fe} / \mathrm{H}] \sim$ 0.12 ) and $E_{(b-y)}=0.071$. Both are close to the values derived (Sect. 3.3) using our membership information and data from earlier studies (Anthony-Twarog \& Twarog 1987; Nissen 1988). The resulting age range is 1.6$1.8 \mathrm{Gyr}$, leading to a best estimate for the age of IC 4651 of $1.7 \pm 0.15 \mathrm{Gyr}$, not including systematic effects in the models. IC 4651 therefore appears to be slightly older than its sister cluster NGC 3680, by $\sim 0.1-0.2$ Gyr.

\section{Dynamical evolution of IC 4651}

Mass segregation, i.e. increasing central concentration of progressively more massive stars (Spitzer \& Mathieu 1987 ), is seen in several open clusters (see e.g. Raboud \& Mermilliod 1998a; Nordström et al. 1997; Mathieu 1985). On average, binary systems are more massive than single stars. It is therefore expected to find binaries, together with the most massive single stars, to be concentrated towards the center of a relaxed cluster. If IC 4651 is older than its own relaxation time, the currently accepted dynamical models predict the appearance of such segregation of stellar masses; see, e.g, de la Fuente Marcos (1997); Portegies Zwart et al. (2001).

\subsection{Test for mass-segregation in IC 4651}

Our photometry and radial-velocity data allow us to test for mass segregation in IC 4651. The radial-velocity data unambiguously classify 86 turnoff or giant stars as single or binary cluster members. From their proximity to the well-defined cluster sequence in the $(v-y)$ vs. $V$ CMD, we identify another 652 stars as probable (photometric) members, especially on the lower main sequence.

The coordinates of the cluster center, $(\alpha, \delta)_{2000}=$ $\left(17^{\mathrm{h}} 24^{\mathrm{s}} 50^{\mathrm{s}},-49^{\circ} 57^{\prime} 01^{\prime \prime}\right)$, were determined as the mean of the coordinates for the 86 certified single and binary cluster members. The projected radial distance from the cluster center was then computed for all 738 known and candidate cluster stars. Next, the sample was divided into bright and faint subsamples, corresponding to mass ranges 
of $\sim 0.6 \quad M_{\odot}-1.5 \quad M_{\odot}$ and $\sim 1.5 \quad M_{\odot}-2.1 M_{\odot}$, respectively. The goal was to create two samples with comparable numbers of stars (after field star subtraction), and with the largest possible ratio between the mean masses of the two samples. Dividing between bright and faint stars at $V=13.4$, corresponding to $\sim 1.5 M_{\odot}$, yielded 144 stars on the more massive side and 592 stars in the less massive group, for a ratio between the mean masses of the two samples of 1.89. The observed field was then divided into 15 annuli with radii from $1^{\prime}$ to $15^{\prime}$, in steps of one arcminute.

Using location in the CMD as the only criterion for identifying cluster stars has the drawback of including a fraction of field stars. The contamination by field stars increases with increasing magnitude and becomes significant on the main sequence below $\sim 15^{\mathrm{m}}$.

In order to estimate the radial density distribution of field stars both brighter and fainter than $V=15^{\mathrm{m}}$, we calculated the distance to the cluster center of field stars in selected areas above and below the cluster sequence in the CMD. Figure 9 shows the radial density distribution for the field stars above (solid) and below (dotted) the lower cluster sequence $\left(V=15^{\mathrm{m}}-18^{\mathrm{m}}\right)$. The mean density levels of the two distributions are indicated by horizontal lines. In order to estimate the density of field stars on the lower cluster sequence itself we calculated the average of the two mean levels, $\sim 0.75$ star per square arcminute (dashed line). The same mean density levels were obtained by dividing the total number of stars in each of the two test groups by the total area of the observed field. A similar estimate of the field contamination on the upper cluster sequence $\left(V<15^{\mathrm{m}}\right)$ resulted in a field star density of $\sim 0.08$ star per square arcminute.

Note that the method of counting field stars adjacent to the cluster sequence will inevitably include some cluster binaries above the sequence and possibly also some cluster stars scattered into the area below the cluster sequence due to photometric errors in these faint stars. Correcting for these contributions would lead to a even lower estimate of the field star density. For the moment, however, we adopt a field density of 0.08 stars per square arcminute for $V<15^{\mathrm{m}} 0$ and 0.75 stars per square arcminute for $V>15^{\mathrm{m}} 0$.

Figure 10 shows the radial density distribution of the certain (radial-velocity) and probable (photometric) cluster members. The cluster star densities do not fall to the estimated field star levels, even at the edge of the observed field. This is especially striking for the lower main sequence, but true also for the brighter stars, showing that even the field observed in the present study was not sufficient to cover the entire cluster: Additional cluster stars, both faint and bright, are very likely still to be found outside the currently known field of IC 4651.

After subtraction of the estimated field star contributions (see Fig. 10), the total numbers of stars in the highand low-mass samples defined previously (i.e. brighter and fainter than $V=13.4$ ) are 110 and 228, respectively.

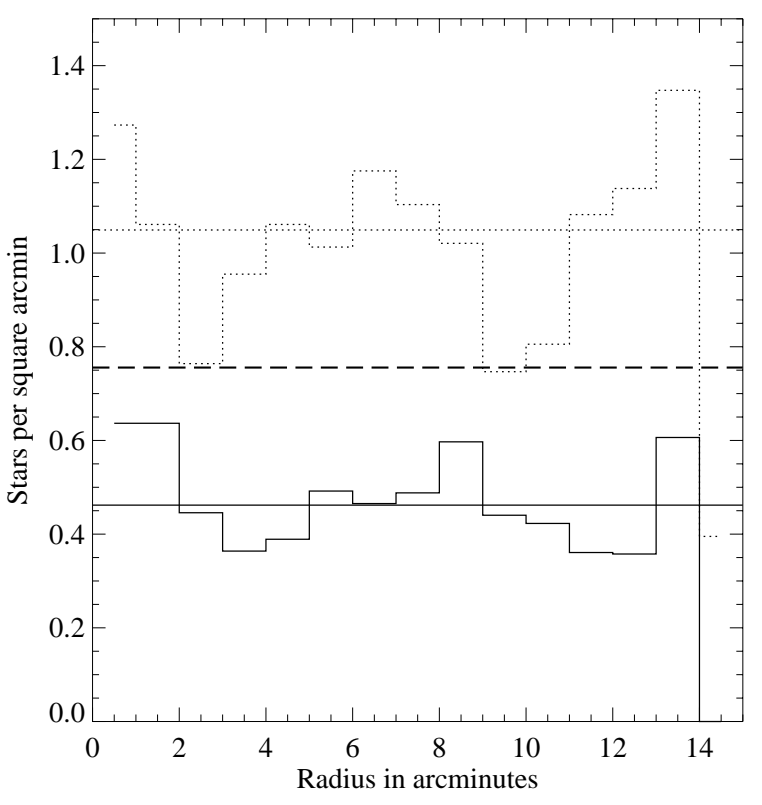

Fig. 9. Radial density distribution of field stars just above (solid) and below (dotted) the lower main sequence of IC 4651 $\left(15^{\mathrm{m}}<V<18^{\mathrm{m}}\right)$. The horizontal lines (coded as the corresponding histograms) indicate the mean densities of the two distributions. The horizontal dashed line shows the average of the two mean values.

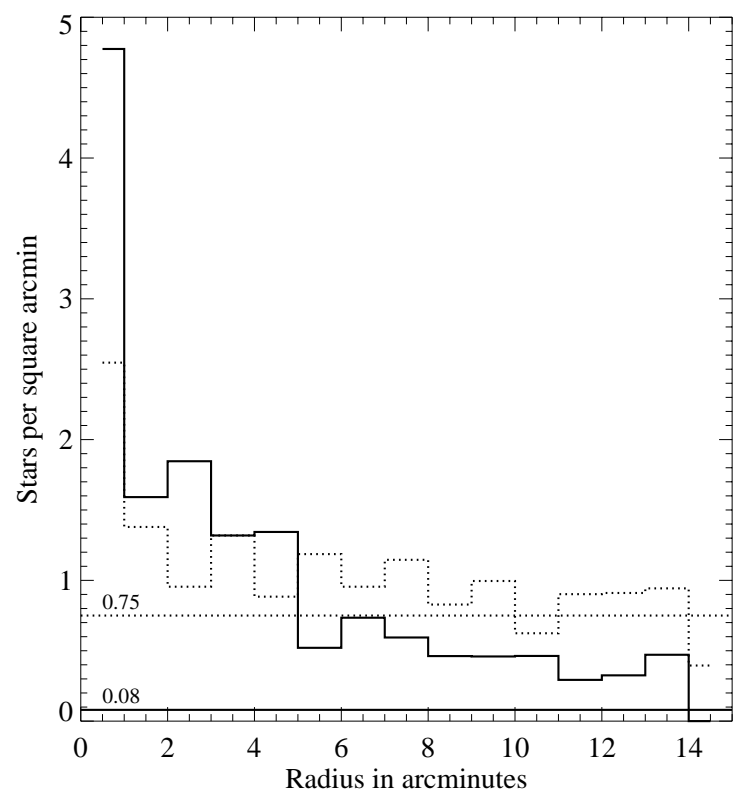

Fig. 10. Radial density distributions of the bright and faint certain and probable cluster members. The solid histogram represents the bright sample $\left(V<15.0^{\mathrm{m}} ; M \gtrsim 1.2 M_{\odot}\right)$, the dotted histogram the faint sample $\left(V>15.0^{\mathrm{m}}\right)$. The matching horizontal lines show the estimated field star densities in each sample (see text).

The normalized and field-subtracted radial density distributions of these two samples are shown in Fig. 11.

A $\chi^{2}$ test was applied to test for mass segregation in IC 4651. Because the field star levels were determined without use of the radial density distributions, all radial 


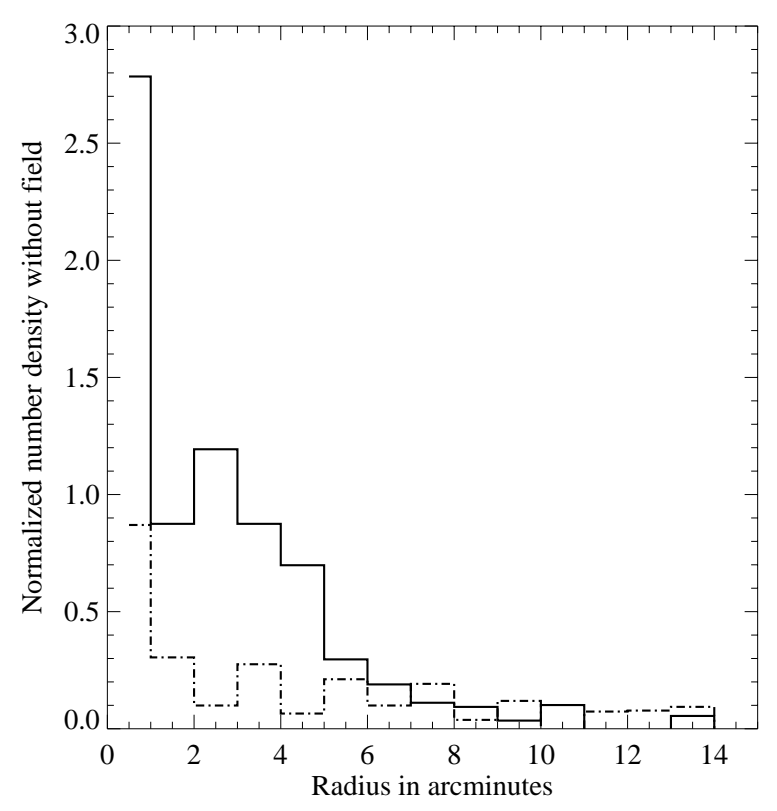

Fig. 11. Normalized and field-subtracted radial density distribution of the certain and probable cluster members. The solid histogram represents the high-mass stars $\left(M \gtrsim 1.5 M_{\odot}, V<\right.$ 13.4), the dashed histogram the lower-mass sample $(M \lesssim$ $\left.1.5 M_{\odot}, V>13.4\right)$.

bins out to $14^{\prime}$ were used in the test. The resultant probability that cluster stars with masses greater and smaller than $\sim 1.5 M_{\odot}$ are not drawn from the same parent radial distribution is $96.4 \%$. It is indeed noticeable in Fig. 11 that nearly all the turn-off and red giant stars are located at radii smaller than $\sim 7^{\prime}$. However, the derived formal probability is quite sensitive to the applied field star correction and the magnitude chosen to separate the two samples. Overall, we conclude that our data do provide moderately strong evidence for mass segregation in IC 4651.

This investigation of the radial density distribution of cluster and field stars, together with the perfect match of the isochrones to the faintest parts of the cluster sequence, indicates a need for an even deeper photometric study of IC 4651 in an even larger field. A larger field should add many faint, but also some bright stars to the membership list and also provide a better estimate of the field star densities from observations at larger radii. This, in turn, would allow to define better the true richness and size of IC 4651, as well as further improve the definition of the lower cluster main sequence. Photometric observations in the Johnson $V$ and $I$ bands covering a large field $\left(2^{\circ} \times\right.$ $2^{\circ}$ ) around the cluster have already been obtained by the authors; it would be most interesting to complement these with a comprehensive radial-velocity survey using a multifiber instrument. If these data were to show that the true size of this "well-known" cluster is even larger than the factor of two relative to the classical literature value which we have already found, then one might have serious doubts about the current catalogue values for other, less wellstudied clusters.

\subsection{Mass function}

Based on the relation between stellar mass and $V$ magnitude for the best-fit Geneva and Yale 2000 isochrones, masses for individual stars were derived. Fifteen brightness intervals were used to divide the cluster mass range from $\sim 0.4 M_{\odot}$ to $\sim 2.0 M_{\odot}$ into mass intervals with a roughly constant width of $0.1 M_{\odot}$. Single radial-velocity members are then simply assigned a mass according to their $V$ magnitude.

Decomposition of binaries. Stars identified as binaries from the radial-velocity data (except MEI 11507, a possible blue straggler) were decomposed into single stars in the following way: Assuming a mass-luminosity relation of $L \propto M^{3}$ on the upper main-sequence (ChristensenDalsgaard 1993), a displacement of $\sim-0.1$ in $V$ from the best-fit isochrone corresponds to a mass ratio between the primary and secondary component of $\sim 2$. Therefore, binaries located within $\sim 0.1$ from the isochrone are assumed to consist of a primary component with a mass corresponding to that of the isochrone, and a secondary component with half that mass.

For each of the 13 binaries located more than $\sim 0.1^{\mathrm{m}}$ above the best-fit isochrone (MEI 7753, 6725, 6726, 11670, 7309, 9278, 14364, 8302, 11507, 5658, 8080, 9357, 11504; see Fig. 3a), we assumed a series of possible secondary components on the lower main sequence. Next, corresponding primary component loci in the CMD were computed so as to match the observed location of the binary. The intersection between the track for the primary component and the cluster sequence then fixes the brightness and therefore the mass of both the primary and secondary star. Figure 12 shows an example of such a primary track for the binary MEI 6725 .

By the procedures illustrated above, the 42 known binary systems were decomposed into single stars. Figure 13 shows the mass function of the individual stars resulting from the decomposition plus the single stars identified by radial velocity measurements. The estimated total mass contribution from this group of cluster stars is $\sim 166 M_{\odot}$.

The many $(\sim 650)$ new cluster stars found at all magnitudes in our CCD photometry have no observational data on membership and duplicity as yet. Nevertheless, they more than double the number of cluster members in the turnoff region, and the entire low-mass cluster population is a new discovery. Their contribution to the total cluster mass and mass function is therefore important and cannot be neglected. The method we have used to estimate masses for these "new" photometric cluster stars is similar to that used for the established radial-velocity members.

The number of field stars in each mass bin was determined using the method described in Sect. 5.1: in each brightness interval (corresponding to a mass interval), stars were counted in two areas bordering the cluster sequence. The mean density of stars was used to estimate the number of field stars overlapping the cluster sequence. As explained above, this method is likely to slightly 


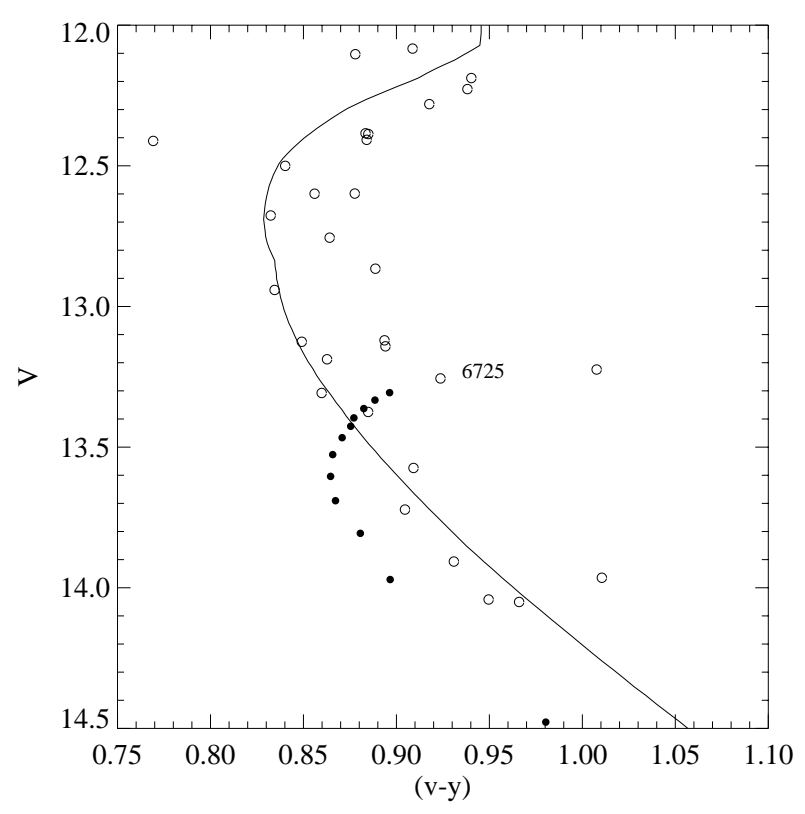

Fig. 12. Binary stars (circles) near the turn-off of IC 4651 together with the best-fit Geneva isochrone. For a sequence of assumed lower main-sequence secondaries, the filled circles show the locus of the matching primary components reproducing the observed position of the binary system MEI 6725. The primary component on the isochrone has a mass of $1.45 M_{\odot}$, the corresponding secondary star a mass of $1.15 M_{\odot}$.

overestimate the field star density. After field subtraction 301 stars were left on the cluster sequence.

Due to mass segregation, however, the binary fraction amongst the outlying stars is probably smaller than the $55 \%$ found in Sect. 3.1 for the turnoff stars in the inner region; we have assumed an average binary fraction of $40 \%$ (120 of the 301 candidate members). We also assume that each binary consists of a primary component with mass corresponding to its location on the isochrone and a secondary component with half that mass. The resulting mass function for the "new" main-sequence stars is shown in Fig. 14. The lack of stars with mass of $\sim 1.1 M_{\odot}$ is due to the effects of binning. The estimated contribution to the cluster mass from the 421 individual stars (181 single stars, 120 primary components, and 120 secondary components) is $\sim 463 M_{\odot}$.

Summing the mass contributions from the two groups of stars with and without radial velocity measurements, we estimate the present total mass of IC 4651 within our $\sim 21^{\prime} \times 21^{\prime}$ field to be $(166+463) M_{\odot} \simeq 630 M_{\odot}$.

With masses now estimated for every plausible cluster star, the dynamical state of IC 4651 can be discussed in more quantitative terms. Figure 15 shows the overall present-day mass function for IC 4651. The open histogram includes the masses of both single stars and individual components of decomposed binary systems. The shaded histogram shows the subsample of stars with radial velocity measurements. For comparison, we also show the three different initial mass functions (IMFs) by Salpeter (1955, solid), Miller \& Scalo (1979, dotted) and

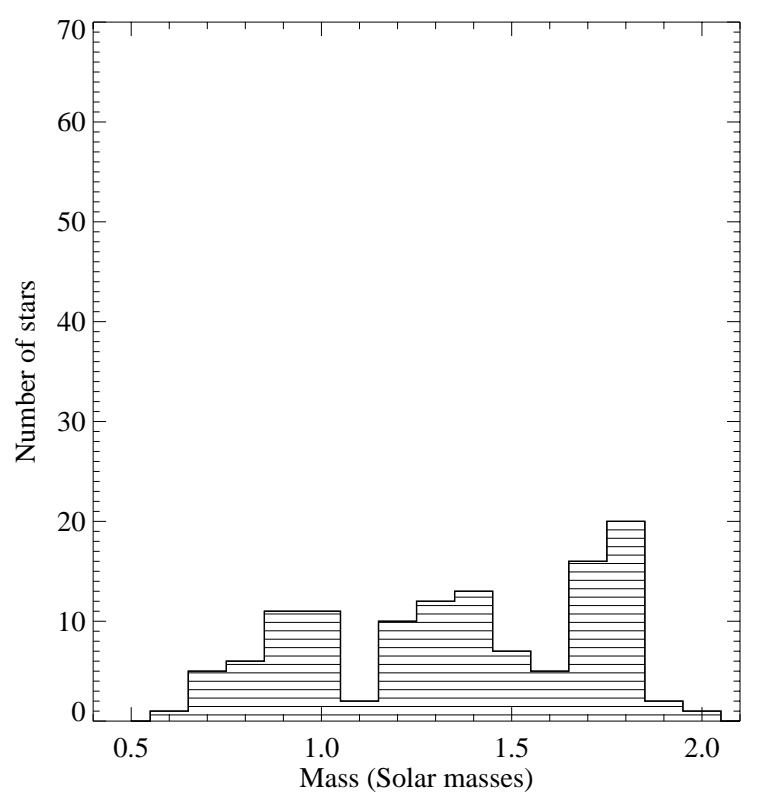

Fig. 13. The mass function of the single members and the components of the 42 decomposed binaries.

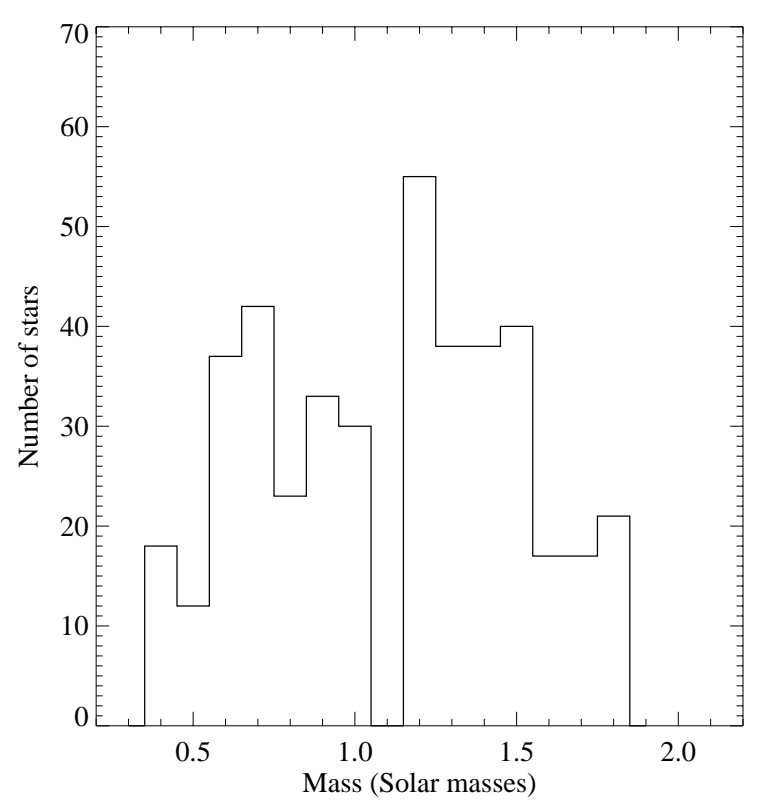

Fig. 14. Mass function of the 421 individual outer-field and lower main-sequence photometric members (181 single stars, 120 primary components, and 120 secondary components).

Kroupa et al. (1993, dashed), normalized to the total number of stars in the range $0.1-60 M_{\odot}$ and scaled to match the number of single stars now observed in the mass bin 1.75-1.85 $M_{\odot}$. Comparing the IMFs with the present-day mass function enables us to estimate the total initial number and mass of stars in IC 4651 as well as the fraction lost during the lifetime of the cluster.

The following results for the initial number of stars and total mass of IC 4651 are obtained: $\left(N_{\text {initial }}, M_{\text {initial }}\right) \simeq\left(28304,9756 M_{\odot}\right),\left(8286,5295 M_{\odot}\right)$, and $\left(15493,6849 M_{\odot}\right)$ for the Salpeter, Miller-Scalo and 


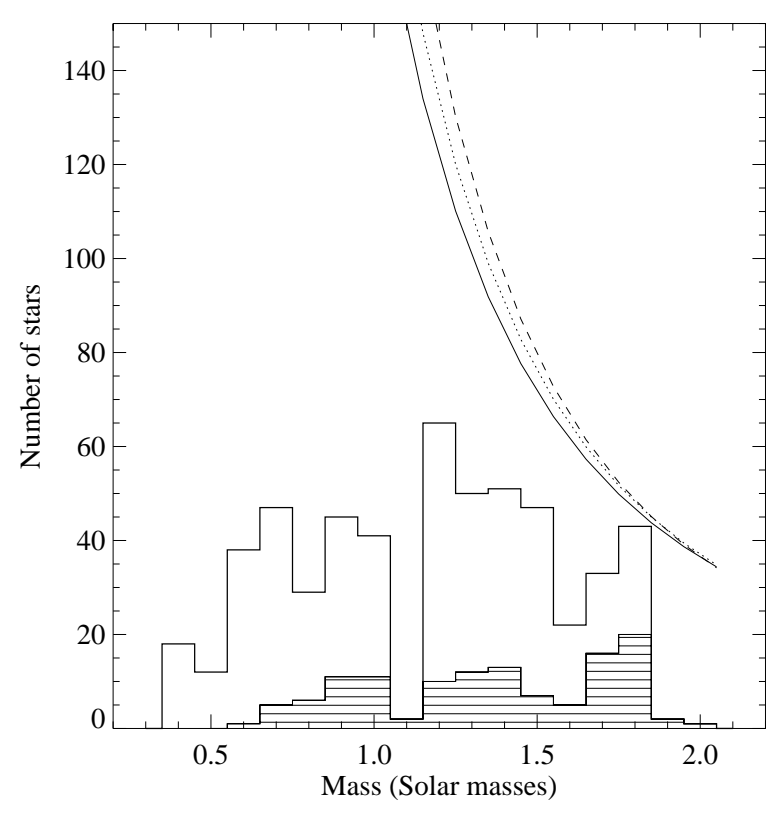

Fig. 15. Overall present-day mass function for IC 4651 together with the IMFs by Salpeter (solid), Miller \& Scalo (dotted) and Kroupa et al. (dashed), normalized to match the 1.75-1.85 $M_{\odot}$ bin. The shaded histogram shows the part of the sample with radial-velocity data.

Kroupa et al. IMFs, respectively. Adopting the least extreme Miller-Scalo IMF (which Padoan et al. 1996 support from both theory and observations), we find that IC 4651 now contains only $\sim 7 \%$ of its original stars and $\sim 12 \%$ of its initial mass, all computed within the observed field. We note, however, that also stars in the mass range 1.75$1.85 M_{\odot}$ may well have been lost from the cluster. Our estimate of the initial cluster mass as well as the mass lost from the cluster is therefore likely to be somewhat conservative.

Mass loss from the cluster can be due to two different processes: 1) Evolution of high mass stars, and 2) evaporation of low mass stars (dynamical mass loss). We discuss these in turn.

$1)$ : the original, most massive cluster stars in the range $\sim 2 M_{\odot}-\sim 60 M_{\odot}$ have completed their evolution to white dwarf or neutron star remnants. The above IMFs predict that IC 4651 initially contained 308-490 stars in the mass range 2-60 $M_{\odot}$. Adopting the Miller-Scalo IMF, a total of $\sim 1871 M_{\odot}$ or $\sim 35 \%$ of the initial cluster mass has thus been lost due to evolution of the $\sim 430$ most massive stars in the young IC 4651 ( $~ 5 \%$ of the initial population)

Nordström et al. (1997) also found that NGC 3680 has lost $\sim 30 \%$ of its initial mass due to evolution of its high mass stars. This similarity is indeed expected because the ages and turn-off masses $\left(\sim 1.7 M_{\odot}\right)$ of the two clusters are nearly identical. We note that both in IC 4651 and NGC 3680, a substantial fraction of the high-mass component might still be present in the form of stellar remnants, primarily white dwarfs. Such white dwarfs are expected to be bright enough to remain observable with medium-sized telescopes.
2): low-mass stars are lost from the central regions of the cluster due to mass segregation, and again from the outer parts due to galactic tidal effects (de la Fuente Marcos 1997; Terlevich 1987). Formation of an outer halo of low-mass stars at 1-2 tidal radii from the cluster center (Terlevich 1987) causes the outermost stars to dynamically evaporate from the cluster. In IC 4651, many of the newly discovered lower main-sequence stars are located outside the central field observed in previous studies. Above, we have found indications of moderate mass segregation (see Sect. 5.1), and we have also shown that additional cluster stars are likely to be found at even greater distances from IC 4651 than those covered in our photometric survey field, the radius of which is less than half the estimated tidal radius of IC $4651\left(\sim 22^{\prime}\right)$. Further studies may succeed in mapping the outer halo of IC 4651 and define its true radial extent.

From the information available within our observed fields, IC 4651 appears to have lost $\sim 53 \%$ of its original mass due to evaporation of low-mass stars $\left(0.1 M_{\odot} \lesssim M \lesssim 2.0 M_{\odot}\right)$, corresponding to $\gtrsim 88 \%$ of the initial number of stars. For comparison, NGC 3680 was found to have lost $\gtrsim 90 \%$ of its original low-mass stars (Nordström et al. 1997), containing $\sim 60 \%$ of the cluster mass within the central $13^{\prime} \times 13^{\prime}$.

\subsection{Galactic environment of IC 4651}

Our "twin" clusters IC 4651 and NGC 3680 are almost identical in age, turn-off mass, and metallicity. They differ strongly, on the other hand, in present (and perhaps also initial) stellar population and total mass. Both clusters have undergone significant dynamical evolution, but NGC 3680 is clearly in a far more advanced stage than IC 4651, judged by our results on mass segregation and evaporation of low-mass stars. In fact, if significant numbers of stars of all masses were lost from NGC 3680 in this process, the two clusters could have been even more similar at birth.

As with other twins, it is natural to search for an explanation of these differences in terms of environmental effects: theory (e.g. Terlevich 1987) suggests that a single close encounter with a massive object may completely disrupt an open cluster in about $10^{8} \mathrm{yr}$. Depending on their orbits in the Galactic disk, both clusters will have been exposed to close passages of giant molecular clouds or other massive objects which may cause tidal stripping of their low mass stars, and indeed Friel (1995) demonstrated that no open clusters older than the Hyades have survived significantly closer to the Galactic center than the Sun.

The galactic positions of the two clusters are: $(l, b)=$ $\left(340.1^{\circ},-7.9^{\circ}\right)$ and $r=1.0 \mathrm{kpc}$ for IC 4651 ; and $(l, b)=$ $\left(286.8^{\circ},+16.9^{\circ}\right)$ and $r=1.3 \mathrm{kpc}$ for NGC 3680 . IC 4651 is thus closer to both the galactic plane and the galactic center than NGC 3680 and would naively be expected to be more, not less dynamically evolved than the latter as we observe. 
Table 3. Space motions $\left(\mathrm{km} \mathrm{s}^{-1}\right)$ and Galactic orbital elements (kpc except for $e$ ) for IC 4651 and NGC $3680 ; R_{0}=8 \mathrm{kpc}$ is assumed.

\begin{tabular}{lrrrrrrr}
\hline \hline Cluster & $U$ & $V$ & $W$ & $R_{\min }$ & $R_{\max }$ & $z_{\max }$ & $e$ \\
\hline IC 4651 & -22 & 6 & 4 & 7.0 & 10.2 & 0.20 & 0.19 \\
NGC 3680 & -22 & -10 & -7 & 7.2 & 8.3 & 0.41 & 0.07 \\
\hline
\end{tabular}

In order to explore whether the current positions of the two clusters are a good guide to their dynamical histories, the brightest stars in both were identified in the TYCHO2 catalogue (Høg et al. 2000). Average absolute proper motions were computed to be $\left(\mu_{\alpha}, \mu_{\delta}\right)=(0,-2)$ mas yr $^{-1}$ (IC 4651, 6 stars) and $\left(\mu_{\alpha}, \mu_{\delta}\right)=(-6,0)$ mas yr $^{-1}$

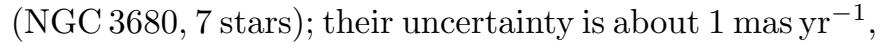
depending mostly on which outlying points are eliminated. From these and their known positions, distances, and mean radial velocities, the space motions given in Table 3 follow. From these in turn, the orbital motions were integrated backwards for 1.6 Gyr in the Dehnen \& Binney (1998) axisymmetric Galactic potential (their Model 2, assuming a Solar galactocentric distance of $R_{0}=8 \mathrm{kpc}$ ). The resulting orbital parameters are also listed in Table 3. Uncertainties in $(U, V, W)$ due to errors in proper motions, distances, and radial velocities are evaluated to be of the order of $2 \mathrm{~km} \mathrm{~s}^{-1}$, in the orbital parameters about $0.1 \mathrm{kpc}$.

Table 3 reveals a non-trivial result: The mean galactocentric distance of IC 4651 ( $8.6 \mathrm{kpc})$ is in fact larger than that of NGC $3680(7.7 \mathrm{kpc})$ despite the fact that they happen to lie in the reverse order at present. Thus, the actual orbits of the two clusters provide a natural explanation why NGC 3680 is the more evolved of the two, a situation that appeared puzzling when only a snapshot of their present locations was considered. A further step, which we have not taken, would be to follow these orbits backwards in time and search for any close passages of massive objects in the disk - a task which would also require building an inventory of such objects and their velocities.

\section{Conclusions}

Our new Strömgren uvby photometry and long-term radial velocity measurements have substantially deepened our understanding of IC 4651. The detailed analysis presented in this paper places IC 4651 in the small company of open star clusters for which data of similar detail and completeness exist (i.e., the Hyades, Pleiades, Praesepe, NGC 3680, and NGC 6231).

The present study has radically revised previous views of IC 4651 as regards mass, stellar population, and size. In addition, our understanding of its present structure and dynamical state has been greatly improved. Together with NGC 3680 (Nordström et al. 1997), IC 4651 now provides an important benchmark for studies of the dynamical evolution of open star clusters.
Our main results on IC 4651 can be summarized as follows:

1) IC 4651 is at least twice as rich in stars as previously thought, and the main sequence is well defined to $V \sim 18^{\mathrm{m}}$.

2) From precise radial-velocity data for 104 stars brighter than $V \sim 14.5$, the membership and duplicity of these stars have been investigated. In total, 86 (80\%) of the 104 stars are found to be cluster members; of these, $42(49 \%)$ are spectroscopic binaries. 5 stars are classified as "possible members", and 13 (13\%) are field stars. Thus, at least for the brighter sample of cluster stars, the frequency of binaries is high (similar to NGC 3680, Nordström et al. 1997), while field star contamination is relatively unimportant.

From 12 single red-giant and 32 single F-type mainsequence members we determine a mean cluster radial velocity of $-30.76 \pm 0.20 \mathrm{~km} \mathrm{~s}^{-1}$. The internal radialvelocity dispersion as determined from the single giants and corrected for observational errors, is $0.74 \mathrm{~km} \mathrm{~s}^{-1}$.

The single main-sequence stars in IC 4651 have a mean rotational velocity of $18.8 \pm 2.4 \mathrm{~km} \mathrm{~s}^{-1}$ (standard error of the mean), with a standard deviation of $13.1 \mathrm{~km} \mathrm{~s}^{-1}$.

3) Using only single member stars measured by Nissen (1988), and Anthony-Twarog \& Twarog (1987) and calculating the metallicity-index $\left(m_{1}\right)$ from the new uvby data, the reddening and metallicity of IC 4651 were found to be $E_{(b-y)}=0.076 \pm 0.012$ and $[\mathrm{Fe} / \mathrm{H}]=0.13 \pm 0.05$. The latter is in good agreement with the recent preliminary spectroscopic determination by Bragaglia et al. (2001). The distance as determined from a direct fit to the Hyades main sequence is $1.01 \pm 0.05 \mathrm{kpc}$.

4) Two sets of stellar evolution models fitted to the single cluster members in the turn-off show that overshooting from the convective cores is significant in IC 4651. The preferred models have $[\mathrm{Fe} / \mathrm{H}] \sim 0.12$ (Hyades) and are fit for $E_{(b-y)}=0.071$. We estimate the age of IC 4651 to be in the range 1.6-1.8 Gyr, with a mean value of $1.7 \pm 0.15 \mathrm{Gyr}$.

5) From the best-fit isochrone, we have assigned individual masses to all stars in IC 4651; the mass at the tip of the turn-off is $\sim 1.75 M_{\odot}$. Binaries were decomposed into their primary and secondary components, and the contribution to the mass function from the secondary components of known spectroscopic binaries as well as suspected binaries in the outer field was estimated. The current total mass of IC 4651 within our field of $\sim 21^{\prime} \times 21^{\prime}$ is estimated to be $\sim 630 M_{\odot}$, leading to an estimated tidal cutoff radius of $\sim 22^{\prime}$.

6) The initial stellar population and total mass of the cluster are estimated by fitting three initial mass functions to the present-day mass function. Using the least extreme IMF (Miller-Scalo), IC 4651 is estimated to have contained $\sim 8300$ stars initially, with a total mass of $\sim 5300 M_{\odot}$. Thus, only $\sim 7 \%$ of the original cluster stars and $\sim 12 \%$ of the initial mass now remain within the field studied. 
Of the initial cluster mass, about $\sim 35 \%\left(1900 M_{\odot}\right)$ has been lost due to the evolution of the most massive stars, while $\sim 53 \%$ has been lost dynamically, corresponding to $\sim 88 \%$ of the initial population of low mass cluster stars. Further studies may reveal how large a fraction of the low mass stars may remain gravitationally bound in an extended halo around IC 4651. The spatial distribution of the cluster members and field stars strongly suggests that enlarging the observed field should lead to the detection of even more distant cluster stars; the field studied so far still only reaches about half the estimated tidal radius.

7) Computing the Galactic orbits of both clusters reveals that IC 4651 is, on average, more distant from the Galactic center than NGC 3680, although the opposite is true for their present locations. This may provide a natural explanation why NGC 3680 is dynamically more evolved than IC 4651 despite their identical ages.

Acknowledgements. We thank Michael I. Andersen and Frank Grundahl for help in planning and performing the photometric observations and data reduction, and Stephane Udry and Michel Mayor for reducing the CORAVEL observations in Geneva. We are much indebted to Dr. Johan Holmberg, Lund Observatory, for computing the space motions and Galactic orbits discussed in Sect. 5.3. Finally, we thank Robert D. Mathieu and Sydney Barnes for helpful discussions and comments and Sukyoung Yi for providing the new Yale isochrones.

This study has been supported by the Danish Natural Science Research Council via its Ground Based Astronomical Instrument Center, and by the US National Science Foundation under grant No. AST 9731302 (R. D. Mathieu, P.I.). S. Meibom gratefully acknowledges a Ph.D. fellowship from "Forskeruddannelsesrådet" (The Danish Research Academy). B. Nordström thanks the Carlsberg Foundation and the Swedish Research Council for financial support.

\section{References}

Anthony-Twarog, B. J., \& Twarog, B. A. 1987, AJ, 94, 1222

Anthony-Twarog, B. J., Mukherjee, K., Caldwell, N., et al. 1988, AJ, 95, 1453

Anthony-Twarog, B. J., \& Twarog, B. A. 2000, AJ, 119, 2282

Benz, W., \& Mayor, M. 1981, A\&A, 93, 235

Benz, W., \& Mayor, M. 1984, A\&A, 138, 183

Bragaglia, A., Tosi, M., Carretta, E., \& Gratton, R. G. 2001, in Cosmic Evolution, ed. E. Vangioni-Flam, R. Ferlet, \& M. Lemoine (World Scientific, Singapore), 209

Christensen-Dalsgaard, J. 1993, Stellar structure and evolution, Lecture notes, Aarhus University

Crawford, D. L., \& Mandwewala, N. 1976, PASP, 88, 917

Daniel, S. A., Latham, D. W., Mathieu, R. L., \& Twarog, B. A. 1994, PASP, 106, 281

Dehnen, W., \& Binney, J. J. 1998, MNRAS, 294, 429

de la Fuente Marcos, R. 1997, A\&A, 322, 764

Edvardsson, B., Andersen, J., Gustafsson, B., et al. 1993, A\&A, 275, 101

Eggen, O. J. 1971, ApJ, 166, 87

Friel, E. D., \& Janes, K. A. 1993, A\&A, 267, 75

Friel, E. D. 1995, ARA\&A, 33, 381

Giménez, A., Guinan, E. F., \& Montesinos, B., (eds.) 1999, Theory and Tests of Convection in Stellar Structure, ASP. Conf. Ser., 173
Green, E. M., Demarque, P., \& King, C. R. 1987, in The Revised Yale Isochrones and Luminosity Functions (Yale Univ. Obs., New Haven)

Høg, E., Fabricius, C., Makarov, V. V., et al. 2000, A\&A, 355,27

Iglesias, C. A., Rogers, F. J., \& Wilson, B. G., 1992, ApJ, 397, 717

Iglesias, C. A., \& Rogers, F. J. 1992, ApJ, 464, 943

Janes, K. A., \& Phelps, R. 1994, AJ, 108, 1773

Kjeldsen, H., \& Frandsen, S. 1991, A\&AS, 87, 119

Kozhurina-Platais, V., Demarque, P., Platais, I., Orosz, J. A., \& Barnes, S. 1997, AJ, 113, 1045

Kroupa, P., Tout, C. A., \& Gilmore, G. 1993, MNRAS, 262, 545

Lindoff, U. 1972, A\&AS, 7, 231

Lejeune, Th., Cuisinier, F., \& Buser, R. 1998, A\&A, 130, 65

Maeder, A. 1990, in Astrophysical Ages and Dating Methods, ed. E. Vangioni-Flam, M. Cassé, J. Audouze, \& J. Tran Thanh Van (Eds. Frontières, Gif-sur-Yvette), 71

Mayor, M. 1985, in Stellar Radial Velocities, IAU Colloq. 88, ed. A. G. D. Philip, \& D. W. Latham (L. Davis Press, Schenectady), 35

Mathieu, R. D., Latham, D. W., Griffin, R. F., \& Gunn, J. E. 1986, AJ, 92, 1100

Mathieu, R. D. 1985, in Dynamics of Star Clusters (IAU Symp. 113), ed. J. Goodman, \& P. Hut (D. Reidel, Dordrecht), 427

Mazzei, P., \& Pigatto, L. 1988, A\&A, 193, 148

Meibom, S. 2000, A\&A, 361, 929

Mermilliod, J.-C., Andersen, J., Nordström, B., et al. 1995, A\&A, 299, 53

Miller, G. E., \& Scalo, J. M. 1979, ApJS, 41, 413

Nissen, P. E., Twarog, B. A., \& Crawford, D. L. 1987, AJ, 93, 634

Nissen, P. E. 1988, A\&A, 199, 146

Nordström, B., Andersen, J., \& Andersen, M. I. 1996, A\&AS, 118,407

Nordström, B., Andersen, J., \& Andersen, M. I. 1997, A\&A, 322,460

Olsen, E. H. 1984, A\&AS, 57, 443

Padoan, P., Nordlund, Å., \& Jones, B. J. T. 1996, MNRAS, 288, 145

Pasquini, L., Randich, S., \& Pallavicini, R. 2001, A\&A, 374, 1017

Perryman, M. A. C., Brown, A. G. A., Lebreton, Y., et al. 1998, A\&A, 331, 81

Portegies Zwart, S. F., McMillan, S. L. W., Hut, P., et al. 2001, MNRAS, 321, 199

Raboud, D., \& Mermilliod, J.-C. 1998a, A\&A, 329, 101

Raboud, D., \& Mermilliod, J.-C. 1998b, A\&A, 333, 897

Rogers, F. J., Swenson, F. J., \& Iglesias, C. A. 1996, ApJ, 456, 902

Salpeter, E. E. 1955, ApJ, 121, 161

Schaerer, D., Charbonnel, C., Meynet, G., et al., 1993, A\&AS, 96, 269

Schaller, G., Schaerer, D., Meynet, G., et al. 1992, A\&AS, 96, 269

Spitzer, L., \& Mathieu, R. D. 1987, ApJ, 241, 618

Terlevich, E. 1987, MNRAS, 224, 193

Udry, S., Mayor, M., \& Queloz, D. 1999, in Precise Stellar Radial Velocities, IAU Colloq. 170, ed. J. B. Hearnshaw, \& C. D. Scarfe, ASP Conf. Ser., 185, 367

Wilson, O. C. 1941, ApJ, 93, 29

Yi, S., Demarque, P., Kim, Y.-C., et al. 2002, ApJ, in press 